\title{
Positive solution of a system of integral equations with applications to boundary value problems of differential equations
}

\author{
Chunfang Shen', Hui Zhou' and Liu Yang ${ }^{1,2^{*}}$
}

\section{"Correspondence:}

xjiangfeng@163.com

${ }^{1}$ College of Mathematics and

Statistics, Hefei Normal University,

Hefei, 230061, P.R. China

${ }^{2}$ College of Mathematical Science,

University of Science and

Technology of China, Hefei, 230000,

P.R. China

\begin{abstract}
In this paper, by using the Guo-Krasnoselskii theorem, we investigate the existence and nonexistence of positive solutions of a system of integral equation with parameters which can be seen as an effective generalization of various types of systems of boundary value problems for differential equation on continuous interval and time scales or fractional differential equations. We give a general approach of positive solutions to cover various systems of boundary value problems in a unified way, which avoids treating these problems on a case-by-case basis. Under some growth conditions imposed on the nonlinear term, we obtain explicit ranges of values of parameters with which the problem has a positive solution and has no positive solution, respectively. By giving some examples, we will show how our results may be applied to consider existence of positive solutions to a variety of system of boundary value problems of differential equations, differential equations on time scales or fractional differential equations.
\end{abstract}

MSC: $45 \mathrm{C} 05 ; 34 \mathrm{~B} 18$

Keywords: positive solution; boundary value problem; integral equation; fixed point; cone

\section{Introduction}

We consider the existence of eigenvalues yielding positive solutions to the system of integral equations

$$
\left(P_{\lambda, \mu, \zeta}\right) \quad \begin{cases}u(t)=\lambda \int_{0}^{1} k_{1}(t, s) f(s, u(s), v(s), w(s)) d s, & 0 \leq t \leq 1, \\ v(t)=\mu \int_{0}^{1} k_{2}(t, s) g(s, u(s), v(s), w(s)) d s, & 0 \leq t \leq 1, \\ w(t)=\zeta \int_{0}^{1} k_{3}(t, s) h(s, u(s), v(s), w(s)) d s, & 0 \leq t \leq 1,\end{cases}
$$

where $\lambda, \mu, \zeta$ are positive numbers and

(H1) $f, g, h \in C\left([0,1] \times R^{+} \times R^{+} \times R^{+}, R^{+}\right)$.

(H2) $k_{1}, k_{2}, k_{3}:[0,1] \times[0,1] \rightarrow R^{+}$are continuous functions and there exist an interval $[\xi, \eta] \subset[0,1]$, positive constants $\gamma_{1}, \gamma_{2}, \gamma_{3}$, and functions $\Phi_{1}, \Phi_{2}, \Phi_{3} \in C\left([0,1], R^{+}\right)$ such that

$$
k_{i}(t, s) \leq \Phi_{i}(s), \quad \text { for }(t, s) \in[0,1] \times[0,1], i=1,2,3,
$$

(c) 2016 Shen et al. This article is distributed under the terms of the Creative Commons Attribution 4.0 International License (http://creativecommons.org/licenses/by/4.0/), which permits unrestricted use, distribution, and reproduction in any medium, provided you give appropriate credit to the original author(s) and the source, provide a link to the Creative Commons license, and indicate if changes were made. 
and

$$
k_{i}(t, s) \geq \gamma_{i} \Phi_{i}(s), \quad \text { for }(t, s) \in[\xi, \eta] \times[0,1], i=1,2,3 .
$$

Here we denote $\gamma=\min \left\{\gamma_{1}, \gamma_{2}, \gamma_{3}\right\}$.

Systems of differential equations or integral equations containing three equations have gained considerable popularity and importance due mainly to their demonstrated applications in widespread fields of science and technology. For example, to describe the development of an infectious disease, compartmental models have been given to separate a population into various classes based on the stages of inflection [1]. The classical SIR model is described by partitioning the population into susceptible, infectious, and recovered individuals, denoted by $S, I, R$, respectively. Assume that the disease incubation period is negligible so that each susceptible individual becomes infectious and later recovers with a permanently or temporarily acquired immunity, then the SIR model is governed by the following system of differential equations:

$$
\left\{\begin{array}{l}
\frac{d S}{d t}=-\beta S(t) I(t)-\mu_{1} S(t)+b, \\
\frac{d I}{d t}=\beta S(t) I(t)-\mu_{2} I(t)-\alpha I(t), \\
\frac{d R}{d t}=\alpha I(t)-\mu_{3} R(t),
\end{array}\right.
$$

where the total population size has been normalized to one and the influx of the susceptible comes from a constant recruitment rate $b$. The death rates for the $S, I, R$ classes are given by $\mu_{1}, \mu_{2}, \mu_{3}$, respectively.

It is well known that the predator-prey model, which was proposed by Volterra in 1926, is one of the basic and important models for the interacting species in both ecology and mathematical ecology due to the fact that the predator-prey interaction is the fundamental structure in population dynamic. Since then, various types of predator-prey models described by differential systems have been proposed and the dynamics of these systems has been considered. For example, Song and Chen [2] proposed the following predator-prey system with stage structure:

$$
\left\{\begin{array}{l}
\frac{d u_{1}}{d t}=\alpha u_{2}(t)-r u_{1}(t)-a e^{-t \tau} u_{2}(t-\tau), \\
\frac{d u_{2}}{d t}=a e^{-t \tau} u_{2}(t-\tau)-m u_{2}^{2}(t)-a_{1} u_{2}(t) v(t), \\
\frac{d v}{d t}=v(t)\left[r_{1}+a_{2} u_{2}(t)-b v(t)\right]
\end{array}\right.
$$

where $u_{1}(t), u_{2}(t)$ represent the densities of immature and mature population of the prey species, respectively, $v(t)$ represent the density of the predator.

Positive solutions of a $n$-dimensional differential equation system or fractional differential equation system with some boundary conditions have received wide attention due to its distinguished applications in engineering, science, mathematical biology and other fields. For $n=1$, see, for example, [3-8] (ordinary differential equations), [9-15] (differential equations on time scales), and [16-20] (fractional differential equations). For $n=2$, see [21-25] (differential equations on time scales), [26-36] (ordinary differential equations), and [37-54] (fractional differential equations) and references along this line. A considerable number of these problems can be formulated as integral equation or integral equation system usually by finding the corresponding Green's function of these problems. Thus the 
integral equation system can be seen naturally as an effective generalization of these types of boundary value problems. The advantage of studying the integral equation system is that we can avoid considering various boundary value problems of differential equations ad hoc.

The aim of this paper is to give a general approach of positive solutions to cover various systems of boundary value problems for differential equation on continuous interval and time scales or fractional differential equations in a unified way, which avoids treating these problems on a case-by-case basis. We consider the existence and nonexistence of positive solutions of integral equation system $\left(P_{\lambda, \mu, \zeta}\right)$ under the conditions $(\mathrm{H} 1)-(\mathrm{H} 2)$ and so the results obtained in this paper may include some known results as a special cases and can be applied to unconsidered boundary value problems which can be formulated as a system of integral equations like $\left(P_{\lambda, \mu, \zeta}\right)$.

Motivated by Webb and Infante $[3,5]$ and Webb and Lan [4], who established new existence results of positive solutions of a Hammerstein integral equation in an unified way, under some growth condition imposed on the nonlinear term, we obtain explicit ranges of values of $\lambda, \mu$, and $\zeta$ with which the problem $\left(P_{\lambda, \mu, \zeta}\right)$ has a positive solution and has no positive solution, respectively. By giving some examples, we will show how our results may be applied to obtain eigenvalues yielding the existence of positive solutions to a variety of system of boundary value problems of differential equations, differential equations on time scales or fractional differential equations.

The main tool used is the following fixed point theorem by Guo and Krasnoselskii [55].

Lemma 1.1 [55] Let E be a Banach space and $K \subset E$ be a cone. Assume $\Omega_{1}, \Omega_{2}$ are open bounded subsets of $E$ with $0 \in \Omega_{1} \subset \bar{\Omega}_{1} \subset \Omega_{2}$, and let

$$
A: K \cap\left(\Omega_{2} \backslash \bar{\Omega}_{1}\right) \rightarrow K
$$

be a completely continuous operator such that

$$
\|A u\| \leq\|u\|, \quad u \in K \cap \partial \Omega_{1}, \quad \text { and } \quad\|A u\| \geq\|u\|, \quad u \in K \cap \partial \Omega_{2}
$$

or

$$
\|A u\| \geq\|u\|, \quad u \in K \cap \partial \Omega_{1}, \quad \text { and } \quad\|A u\| \leq\|u\|, \quad u \in K \cap \partial \Omega_{2},
$$

then $A$ has a fixed point in $K \cap\left(\Omega_{2} \backslash \bar{\Omega}_{1}\right)$.

\section{Existence results of positive solutions}

In this section we shall consider sufficient conditions on $\lambda, \mu, \zeta, f, g$, and $h$ such that a positive solution with respect to a cone for the problem $\left(P_{\lambda, \mu, \zeta}\right)$ exists.

Let the Banach space $X=\{u \in C[0,1]\}$ be endowed with the norm

$$
\|u\|=\sup _{0 \leq t \leq 1}|u(t)|, \quad u \in X
$$

and the Banach space $Y=X \times X \times X$ with the norm

$$
\|(u, v, w)\|_{Y}=\|u\|+\|v\|+\|w\| .
$$


We define the cone $P \subset Y$ by

$$
P=\left\{u \in E \mid u(t) \geq 0, v(t) \geq 0, w(t) \geq 0, \inf _{\xi \leq t \leq \eta}(u(t)+v(t)+w(t)) \geq \gamma\|(u, v, w)\|_{Y}\right\} .
$$

Define the operators $T_{1}: Y \rightarrow X, T_{2}: Y \rightarrow X, T_{3}: Y \rightarrow X$, and $T: Y \rightarrow Y$ by

$$
\begin{aligned}
& T_{1}(u(t), v(t), w(t)):=\lambda \int_{0}^{1} k_{1}(t, s) f(s, u(s), v(s), w(s)) d s, \\
& T_{2}(u(t), v(t), w(t)):=\mu \int_{0}^{1} k_{2}(t, s) g(s, u(s), v(s), w(s)) d s, \\
& T_{3}(u(t), v(t), w(t)):=\zeta \int_{0}^{1} k_{3}(t, s) h(s, u(s), v(s), w(s)) d s,
\end{aligned}
$$

and

$$
T(u, v, w)=\left(T_{1}(u, v, w), T_{2}(u, v, w), T_{2}(u, v, w)\right) .
$$

It is obvious that the fixed points of the operator $T$ are the positive solutions of the problem $\left(P_{\lambda, \mu, \zeta}\right)$.

Lemma 2.1 $T: P \rightarrow P$ is completely continuous.

Proof The operator $T: P \rightarrow Y$ is nonnegative and equicontinuous in view of the nonnegativeness and continuity of functions $k_{1}(t, s), k_{2}(t, s), k_{3}(t, s)$ and $f(t, u, v, w), g(t, u, v, w)$, $h(t, u, v, w)$.

Let $\Omega \subset P$ be bounded. Then there exists a constant $R_{0}>0$ such that $\|(u, v, w)\|_{Y} \leq$ $R_{0},(u, v, w) \in \Omega$. Denote

$$
\begin{aligned}
R= & \max \left\{\max _{0 \leq t \leq 1,(u, v, w) \in \Omega}|f(t, u, v, w)|, \max _{0 \leq t \leq 1,(u, v, w) \in \Omega}|g(t, u, v, w)|,\right. \\
& \left.\max _{0 \leq t \leq 1,(u, v, w) \in \Omega}|h(t, u, v, w)|\right\}+1 .
\end{aligned}
$$

Then for $(u, v, w) \in \Omega$, we have

$$
\begin{aligned}
& \left|T_{1}(u, v, w)\right| \leq \lambda \int_{0}^{1} k_{1}(t, s)|f(s, u(s), v(s), w(s))| d s \leq \lambda R \int_{0}^{1} \Phi_{1}(s) d s \\
& \left|T_{2}(u, v, w)\right| \leq \mu \int_{0}^{1} k_{2}(t, s)|f(s, u(s), v(s), w(s))| d s \leq \mu R \int_{0}^{1} \Phi_{2}(s) d s \\
& \left|T_{3}(u, v, w)\right| \leq \zeta \int_{0}^{1} k_{3}(t, s)|f(s, u(s), v(s), w(s))| d s \leq \zeta R \int_{0}^{1} \Phi_{3}(s) d s .
\end{aligned}
$$

Hence $T(\Omega)$ is bounded.

By means of the Arzela-Ascoli theorem, we see that $T$ is completely continuous. Furthermore, considering

$$
\inf _{\xi \leq t \leq \eta} T_{1}(u, v, w)(t) \geq \gamma_{1} \sup _{t^{\prime} \in[0,1]} T_{1}(u, v, w)\left(t^{\prime}\right)
$$




$$
\begin{aligned}
& \inf _{\xi \leq t \leq \eta} T_{2}(u, v, w)(t) \geq \gamma_{2} \sup _{t^{\prime} \in[0,1]} T_{2}(u, v, w)\left(t^{\prime}\right), \\
& \inf _{\xi \leq t \leq \eta} T_{3}(u, v, w)(t) \geq \gamma_{3} \sup _{t^{\prime} \in[0,1]} T_{3}(u, v, w)\left(t^{\prime}\right) .
\end{aligned}
$$

Hence

$$
\begin{aligned}
& \inf _{\xi \leq t \leq \eta}\left|T_{1}(u, v, w)(t)+T_{2}(u, v, w)(t)+T_{3}(u, v, w)(t)\right| \\
& \quad \geq \inf _{\xi \leq t \leq \eta} T_{1}(u, v, w)(t)+\inf _{\xi \leq t \leq \eta} T_{2}(u, v, w)+\inf _{\xi \leq t \leq \eta} T_{3}(u, v, w)(t) \\
& \quad \geq \gamma_{1}\left\|T_{1}(u, v, w)\right\|+\gamma_{2}\left\|T_{2}(u, v, w)\right\|+\gamma_{3}\left\|T_{3}(u, v, w)\right\| \\
& \quad \geq \gamma\|T(u, v, w)\|_{Y} .
\end{aligned}
$$

Thus, we show that $T: P \rightarrow P$ is a completely continuous operator.

Here we introduce the following extreme limits:

$$
\begin{array}{ll}
f_{0}^{s}=\lim _{u+v+w \rightarrow 0^{+}} \sup \max _{t \in[0,1]} \frac{f(t, u, v, w)}{u+v+w}, & f_{0}^{i}=\lim _{u+v+w \rightarrow 0^{+}} \inf \min _{t \in[\xi, \eta]} \frac{f(t, u, v, w)}{u+v+w}, \\
f_{\infty}^{s}=\lim _{u+v+w \rightarrow \infty} \sup \max _{t \in[0,1]} \frac{f(t, u, v, w)}{u+v+w}, & f_{\infty}^{i}=\lim _{u+v+w \rightarrow \infty} \text { inf } \min _{t \in[\xi, \eta]} \frac{f(t, u, v, w)}{u+v+w}, \\
g_{0}^{s}=\lim _{u+v+w \rightarrow 0^{+}} \sup \max _{t \in[0,1]} \frac{g(t, u, v, w)}{u+v+w}, & g_{0}^{i}=\lim _{u+v+w \rightarrow 0^{+}} \text {inf } \min _{t \in[\xi, \eta} \frac{g(t, u, v, w)}{u+v+w}, \\
g_{\infty}^{s}=\lim _{u+v+w \rightarrow \infty} \sup \max _{t \in[0,1]} \frac{g(t, u, v, w)}{u+v+w}, & g_{\infty}^{i}=\lim _{u+v+w \rightarrow \infty} \inf \min _{t \in[\xi, \eta]} \frac{g(t, u, v, w)}{u+v+w} . \\
h_{0}^{s}=\lim _{u+v+w \rightarrow 0^{+}} \sup \max _{t \in[0,1]} \frac{h(t, u, v, w)}{u+v+w}, & h_{0}^{i}=\lim _{u+v+w \rightarrow 0^{+}} \inf \min _{t \in[\xi, \eta]} \frac{h(t, u, v, w)}{u+v+w}, \\
h_{\infty}^{s}=\lim _{u+v+w \rightarrow \infty} \sup \max _{t \in[0,1]} \frac{h(t, u, v, w)}{u+v+w}, & h_{\infty}^{i}=\lim _{u+v+w \rightarrow \infty} \text { inf } \min _{t \in[\xi, \eta]} \frac{h(t, u, v, w)}{u+v+w} .
\end{array}
$$

Denote the positive constants

$$
\begin{array}{lc}
K_{1}=\frac{b}{\gamma \tilde{A} f_{\infty}^{i}}, & K_{2}=\frac{c}{A f_{0}^{s}}, \quad K_{3}=\frac{a}{\gamma \tilde{B} g_{\infty}^{i}}, \\
K_{4}=\frac{d}{B g_{0}^{s}}, & K_{5}=\frac{1-a-b}{\gamma \tilde{C} h_{\infty}^{i}},
\end{array}
$$

where $f_{0}^{s}, g_{0}^{s}, h_{0}^{s}, f_{\infty}^{i}, g_{\infty}^{i}, h_{\infty}^{i} \in(0, \infty), a \in[0,1], b \in(0,1), c \in[0,1], d \in(0,1)$, and

$$
\begin{array}{ll}
A=\int_{0}^{1} \Phi_{1}(s) d s, \quad B=\int_{0}^{1} \Phi_{2}(s) d s, \quad C=\int_{0}^{1} \Phi_{3}(s) d s, \quad \tilde{A}=\gamma \int_{\xi}^{\eta} \Phi_{1}(s) d s \\
\tilde{B}=\gamma \int_{\xi}^{\eta} \Phi_{2}(s) d s, \quad \tilde{C}=\gamma \int_{\xi}^{\eta} \Phi_{3}(s) d s .
\end{array}
$$

Theorem 2.1 Assume that (H1)-(H2) hold. $f_{0}^{s}, g_{0}^{s}, h_{0}^{s}, f_{\infty}^{i}, g_{\infty}^{i}, h_{\infty}^{i} \in(0, \infty), a \in[0,1], b \in$ $(0,1), c \in[0,1], d \in(0,1), K_{1}<K_{2}, K_{3}<K_{4}$, and $K_{5}<K_{6}$, then for $\lambda \in\left(K_{1}, K_{2}\right), \mu \in\left(K_{3}, K_{4}\right)$, and $\zeta \in\left(K_{5}, K_{6}\right)$, the problem $\left(P_{\lambda, \mu, \zeta}\right)$ has at least one positive solution $(u(t), v(t), w(t)), t \in$ $[0,1]$. 
Proof Let $\lambda \in\left(K_{1}, K_{2}\right), \mu \in\left(K_{3}, K_{4}\right), \zeta \in\left(K_{5}, K_{6}\right)$, and $\varepsilon$ be a positive number such that $f_{\infty}^{i}, g_{\infty}^{i}, h_{\infty}^{i}>\varepsilon$, and

$$
\begin{gathered}
\frac{b}{\gamma \tilde{A}\left(f_{\infty}^{i}-\varepsilon\right)}<\lambda<\frac{c}{A\left(f_{0}^{s}+\varepsilon\right)}, \\
\frac{a}{\gamma \tilde{B}\left(g_{\infty}^{i}-\varepsilon\right)}<\mu<\frac{d}{B\left(g_{0}^{s}+\varepsilon\right)}, \\
\frac{1-a-b}{\gamma \tilde{C}\left(h_{\infty}^{i}-\varepsilon\right)}<\zeta<\frac{1-c-d}{C\left(h_{0}^{s}+\varepsilon\right)} .
\end{gathered}
$$

By condition (H1), there exists $R_{1}>0$ such that for $t \in[0,1], u(t) \geq 0, v(t) \geq 0, w(t) \geq 0$ and $u(t)+v(t)+w(t) \leq R_{1}$,

$$
\begin{aligned}
& f(t, u(t), v(t), w(t)) \leq\left(f_{0}^{s}+\varepsilon\right)(u(t)+v(t)+w(t)), \\
& g(t, u(t), v(t), w(t)) \leq\left(g_{0}^{s}+\varepsilon\right)(u(t)+v(t)+w(t)), \\
& h(t, u(t), v(t), w(t)) \leq\left(h_{0}^{s}+\varepsilon\right)(u(t)+v(t)+w(t)) .
\end{aligned}
$$

We define the set

$$
\Omega_{1}=\left\{(u(t), v(t), w(t)) \in Y,\|(u, v, w)\|_{Y}<R_{1}\right\} .
$$

Let $(u, v, w) \in P \cap \partial \Omega_{1}$,

$$
\begin{aligned}
T_{1}(u, v, w)(t) & =\lambda \int_{0}^{1} k_{1}(t, s) f(s, u(s), v(s), w(s)) d s \\
& \leq \lambda \int_{0}^{1} k_{1}(t, s)\left(f_{0}^{s}+\varepsilon\right)(u(s)+v(s)+w(s)) d s \\
& \leq \lambda\left(f_{0}^{s}+\varepsilon\right) \int_{0}^{1} k_{1}(t, s)(\|u\|+\|v\|+\|w\|) d s \\
& \leq \lambda A\left(f_{0}^{s}+\varepsilon\right)\|(u, v, w)\|_{Y} \\
& \leq c\|(u, v, w)\|_{Y} \\
T_{2}(u, v, w)(t) & =\mu \int_{0}^{1} k_{2}(t, s) g(s, u(s), v(s), w(s)) d s \\
& \leq \mu \int_{0}^{1} k_{2}(t, s)\left(g_{0}^{s}+\varepsilon\right)(u(s)+v(s)+w(s)) d s \\
& \leq \mu\left(g_{0}^{s}+\varepsilon\right) \int_{0}^{1} k_{2}(t, s)(\|u\|+\|v\|+\|w\|) d s \\
& \leq \mu B\left(g_{0}^{s}+\varepsilon\right)\|(u, v, w)\|_{Y} \\
& \leq d\|(u, v, w)\|_{Y}, \\
& \leq \zeta \int_{0}^{1} k_{3}(t, s)\left(h_{0}^{s}+\varepsilon\right)(u(s)+v(s)+w(s)) d s \\
T_{3}(u, v, w)(t) & =\zeta \int_{0}^{1} k_{3}(t, s) h(s, u(s), v(s), w(s)) d s
\end{aligned}
$$




$$
\begin{aligned}
& \leq \zeta\left(h_{0}^{s}+\varepsilon\right) \int_{0}^{1} k_{3}(t, s)(\|u\|+\|v\|+\|w\|) d s \\
& \leq \zeta C\left(h_{0}^{s}+\varepsilon\right)\|(u, v, w)\|_{Y} \\
& \leq(1-c-d)\|(u, v, w)\|_{Y} .
\end{aligned}
$$

Then for $(u, v, w) \in P \cap \partial \Omega_{1}$,

$$
\begin{aligned}
\|T(u, v, w)\|_{Y} & =\left\|T_{1}(u, v, w)\right\|+\left\|T_{2}(u, v, w)\right\|+\left\|T_{3}(u, v, w)\right\| \\
& \leq c\|(u, v, w)\|_{Y}+d\|(u, v, w)\|_{Y}+(1-c-d)\|(u, v, w)\|_{Y} \\
& =\|(u, v, w)\|_{Y} .
\end{aligned}
$$

On the other side, by condition (H1) and the definition of $f_{\infty}^{i}, g_{\infty}^{i}$, and $h_{\infty}^{i}$, there exists $\bar{R}_{2}>0$ such that for $t \in[\xi, \eta], u(t) \geq 0, v(t) \geq 0, w(t) \geq 0$, and $u(t)+v(t)+w(t) \geq \bar{R}_{2}$,

$$
\begin{aligned}
& f(t, u(t), v(t), w(t)) \geq\left(f_{\infty}^{i}-\varepsilon\right)(u(t)+v(t)+w(t)), \\
& g(t, u(t), v(t), w(t)) \geq\left(g_{\infty}^{i}-\varepsilon\right)(u(t)+v(t)+w(t)), \\
& h(t, u(t), v(t), w(t)) \geq\left(h_{\infty}^{i}-\varepsilon\right)(u(t)+v(t)+w(t)) .
\end{aligned}
$$

We consider $R_{2}=\max \left\{2 R_{1}, \bar{R}_{2} / \gamma\right\}$, and we define the set

$$
\Omega_{2}=\left\{(u(t), v(t), w(t)) \in Y,\|(u, v, w)\|_{Y}<R_{2}\right\} .
$$

Let $(u, v, w) \in P \cap \Omega_{2}$, then for $(u, v, w) \in P$ with $\|(u, v, w)\|=R_{2}$, we have

$$
\begin{aligned}
T_{1}(u, v, w)(t) & =\lambda \int_{0}^{1} k_{1}(t, s) f(s, u(s), v(s), w(s)) d s \\
& \geq \lambda \int_{\xi}^{\eta} k_{1}(t, s) f(s, u(s), v(s), w(s)) d s \\
& \geq \lambda \int_{\xi}^{\eta} k_{1}(t, s)\left(f_{\infty}^{i}-\varepsilon\right)(u(s)+v(s)+w(s)) d s \\
& \geq \lambda \gamma \int_{\xi}^{\eta} k_{1}(t, s)\left(f_{\infty}^{i}-\varepsilon\right)\|(u, v, w)\|_{Y} d s \\
& \geq \lambda \gamma \tilde{A}\left(f_{\infty}^{i}-\varepsilon\right)\|(u, v, w)\|_{Y} \\
& \geq b\|(u, v, w)\|_{Y}, \\
T_{2}(u, v, w)(t) & =\mu \int_{0}^{1} k_{2}(t, s) g(s, u(s), v(s), w(s)) d s \\
& \geq \mu \int_{\xi}^{\eta} k_{2}(t, s) g(s, u(s), v(s), w(s)) d s \\
& \geq \mu \int_{\xi}^{\eta} k_{2}(t, s)\left(g_{\infty}^{i}-\varepsilon\right)(u(s)+v(s)+w(s)) d s \\
& \geq \mu \gamma \int_{\xi}^{\eta} k_{2}(t, s)\left(g_{\infty}^{i}-\varepsilon\right)\|(u, v, w)\|_{Y} d s
\end{aligned}
$$




$$
\begin{aligned}
& \geq \mu \gamma \tilde{B}\left(g_{\infty}^{i}-\varepsilon\right)\|(u, v, w)\|_{Y} \\
& \geq a\|(u, v, w)\|_{Y}, \\
T_{3}(u, v, w)(t) & =\zeta \int_{0}^{1} k_{3}(t, s) h(s, u(s), v(s)) d s \\
& \geq \zeta \int_{\xi}^{\eta} k_{3}(t, s) h(s, u(s), v(s), w(s)) d s \\
& \geq \zeta \int_{\xi}^{\eta} k_{3}(t, s)\left(h_{\infty}^{i}-\varepsilon\right)(u(s)+v(s)+w(s)) d s \\
& \geq \zeta \gamma \int_{\xi}^{\eta} k_{3}(t, s)\left(h_{\infty}^{i}-\varepsilon\right)\|(u, v, w)\|_{Y} d s \\
& \geq \zeta \gamma \tilde{C}\left(h_{\infty}^{i}-\varepsilon\right)\|(u, v, w)\|_{Y} \\
& \geq(1-a-b)\|(u, v, w)\|_{Y} .
\end{aligned}
$$

Thus,

$$
\begin{aligned}
\|T(u, v, w)\|_{Y} & =\left\|T_{1}(u, v, w)\right\|+\left\|T_{2}(u, v, w)\right\|+\left\|T_{3}(u, v, w)\right\| \\
& \geq b\|(u, v, w)\|_{Y}+a\|(u, v, w)\|_{Y}+(1-a-b)\|(u, v, w)\|_{Y} \\
& =\|(u, v, w)\|_{Y} .
\end{aligned}
$$

By using Lemma 1.1, $T$ has a fixed point $(u, v, w) \in P \cap\left(\Omega_{2} \backslash \bar{\Omega}_{1}\right)$.

By a similar analysis, we can consider the case that the above limits reach 0 or $\infty$. We give the main results here and the proofs are omitted.

Theorem 2.2 Assume that (H1)-(H2) hold. If $f_{0}^{s}=0, g_{0}^{s}, h_{0}^{s}, f_{\infty}^{i}, g_{\infty}^{i}, h_{\infty}^{i} \in(0, \infty), a \in[0,1]$, $b \in(0,1), c \in[0,1], d \in(0,1), K_{3}<K_{4}$, and $K_{5}<K_{6}$, then for $\lambda \in\left(K_{1}, \infty\right), \mu \in\left(K_{3}, K_{4}\right)$, and $\zeta \in\left(K_{5}, K_{6}\right)$, the problem $\left(P_{\lambda, \mu, \zeta}\right)$ has at least one positive solution $(u(t), v(t), w(t)), t \in[0,1]$.

Theorem 2.3 Assume that (H1)-(H2) hold. If $g_{0}^{s}=0, f_{0}^{s}, h_{0}^{s}, f_{\infty}^{i}, g_{\infty}^{i}, h_{\infty}^{i} \in(0, \infty), a \in[0,1]$, $b \in(0,1), c \in[0,1], d \in(0,1), K_{1}<K_{2}$, and $K_{5}<K_{6}$, then for $\lambda \in\left(K_{1}, K_{2}\right), \mu \in\left(K_{3}, \infty\right)$, and $\zeta \in\left(K_{5}, K_{6}\right)$, the problem $\left(P_{\lambda, \mu, \zeta}\right)$ has at least one positive solution $(u(t), v(t), w(t)), t \in[0,1]$.

Theorem 2.4 Assume that (H1)-(H2) hold. If $h_{0}^{s}=0, f_{0}^{s}, g_{0}^{s}, f_{\infty}^{i}, g_{\infty}^{i}, h_{\infty}^{i} \in(0, \infty), a \in[0,1]$, $b \in(0,1), c \in[0,1], d \in(0,1), K_{1}<K_{2}$, and $K_{3}<K_{4}$, then for $\lambda \in\left(K_{1}, K_{2}\right), \mu \in\left(K_{3}, K_{4}\right)$, and $\zeta \in\left(K_{5}, \infty\right)$, the problem $\left(P_{\lambda, \mu, \zeta}\right)$ has at least one positive solution $(u(t), v(t), w(t)), t \in[0,1]$.

Theorem 2.5 Assume that (H1)-(H2) hold. If $f_{0}^{s}=g_{0}^{s}=0, h_{0}^{s}, f_{\infty}^{i}, g_{\infty}^{i}, h_{\infty}^{i} \in(0, \infty), a \in$ $[0,1], b \in(0,1), c \in[0,1], d \in(0,1), K_{5}<K_{6}$, then for $\lambda \in\left(K_{1}, \infty\right), \mu \in\left(K_{3}, \infty\right)$, and $\zeta \in$ $\left(K_{5}, K_{6}\right)$, the problem $\left(P_{\lambda, \mu, \zeta}\right)$ has at least one positive solution $(u(t), v(t), w(t)), t \in[0,1]$.

Theorem 2.6 Assume that (H1)-(H2) hold. If $f_{0}^{s}=h_{0}^{s}=0, g_{0}^{s}, f_{\infty}^{i}, g_{\infty}^{i}, h_{\infty}^{i} \in(0, \infty), a \in$ $[0,1], b \in(0,1), c \in[0,1], d \in(0,1), K_{3}<K_{4}$, then for $\lambda \in\left(K_{1}, \infty\right), \mu \in\left(K_{3}, K_{4}\right)$ and $\zeta \in$ $\left(K_{5}, \infty\right)$, the problem $\left(P_{\lambda, \mu, \zeta}\right)$ has at least one positive solution $(u(t), v(t), w(t)), t \in[0,1]$. 
Theorem 2.7 Assume that (H1)-(H2) hold. If $g_{0}^{s}=h_{0}^{s}=0, f_{0}^{s}, f_{\infty}^{i}, g_{\infty}^{i}, h_{\infty}^{i} \in(0, \infty), a \in$ $[0,1], b \in(0,1), c \in[0,1], d \in(0,1), K_{1}<K_{2}$, then for $\lambda \in\left(K_{1}, K_{2}\right), \mu \in\left(K_{3}, \infty\right)$ and $\zeta \in$ $\left(K_{5}, \infty\right)$, the problem $\left(P_{\lambda, \mu, \zeta}\right)$ has at least one positive solution $(u(t), v(t), w(t)), t \in[0,1]$.

Theorem 2.8 Assume that (H1)-(H2) hold. If $f_{0}^{s}=g_{0}^{s}=h_{0}^{s}=0, f_{\infty}^{i}, g_{\infty}^{i}, h_{\infty}^{i} \in(0, \infty), a \in$ $[0,1], b \in(0,1), c \in[0,1], d \in(0,1)$, then for $\lambda \in\left(K_{1}, \infty\right), \mu \in\left(K_{3}, \infty\right)$, and $\zeta \in\left(K_{5}, \infty\right)$, the problem $\left(P_{\lambda, \mu, \zeta}\right)$ has at least one positive solution $(u(t), v(t), w(t)), t \in[0,1]$.

Theorem 2.9 Assume that (H1)-(H2) hold. If $f_{\infty}^{i}=\infty, f_{0}^{s}, g_{0}^{s}, h_{0}^{s}, g_{\infty}^{i}, h_{\infty}^{i} \in(0, \infty), a \in$ $[0,1], b \in(0,1), c \in[0,1], d \in(0,1), K_{3}<K_{4}$, and $K_{5}<K_{6}$, then for $\lambda \in\left(0, K_{2}\right), \mu \in\left(K_{3}, K_{4}\right)$, and $\zeta \in\left(K_{5}, K_{6}\right)$, the problem $\left(P_{\lambda, \mu, \zeta}\right)$ has at least one positive solution $(u(t), v(t), w(t)), t \in$ $[0,1]$.

Theorem 2.10 Assume that (H1)-(H2) hold. If $g_{\infty}^{i}=\infty, f_{0}^{s}, g_{0}^{s}, h_{0}^{s}, f_{\infty}^{i}, h_{\infty}^{i} \in(0, \infty), a \in$ $[0,1], b \in(0,1), c \in[0,1], d \in(0,1), K_{1}<K_{2}$, and $K_{5}<K_{6}$, then for $\lambda \in\left(K_{1}, K_{2}\right), \mu \in\left(0, K_{4}\right)$, and $\zeta \in\left(K_{5}, K_{6}\right)$, the problem $\left(P_{\lambda, \mu, \zeta}\right)$ has at least one positive solution $(u(t), v(t), w(t)), t \in$ $[0,1]$.

Theorem 2.11 Assume that (H1)-(H2) hold. If $h_{\infty}^{i}=\infty, f_{0}^{s}, g_{0}^{s}, h_{0}^{s}, f_{\infty}^{i}, g_{\infty}^{i} \in(0, \infty), a \in$ $[0,1], b \in(0,1), c \in[0,1], d \in(0,1), K_{1}<K_{2}$, and $K_{3}<K_{4}$, then for $\lambda \in\left(K_{1}, K_{2}\right), \mu \in\left(K_{3}, K_{4}\right)$, and $\zeta \in\left(0, K_{6}\right)$, the problem $\left(P_{\lambda, \mu, \zeta}\right)$ has at least one positive solution $(u(t), v(t), w(t)), t \in$ $[0,1]$.

Theorem 2.12 Assume that (H1)-(H2) hold. If $f_{\infty}^{i}=g_{\infty}^{i}=\infty, f_{0}^{s}, g_{0}^{s}, h_{0}^{s}, h_{\infty}^{i} \in(0, \infty), a \in$ $[0,1], b \in(0,1), c \in[0,1], d \in(0,1), K_{5}<K_{6}$, then for $\lambda \in\left(0, K_{2}\right), \mu \in\left(0, K_{4}\right)$, and $\zeta \in$ $\left(K_{5}, K_{6}\right)$, the problem $\left(P_{\lambda, \mu, \zeta}\right)$ has at least one positive solution $(u(t), v(t), w(t)), t \in[0,1]$.

Theorem 2.13 Assume that (H1)-(H2) hold. If $f_{\infty}^{i}=h_{\infty}^{i}=\infty, f_{0}^{s}, g_{0}^{s}, h_{0}^{s}, g_{\infty}^{i} \in(0, \infty), a \in$ $[0,1], b \in(0,1), c \in[0,1], d \in(0,1), K_{3}<K_{4}$, then for $\lambda \in\left(0, K_{2}\right), \mu \in\left(K_{3}, K_{4}\right)$, and $\zeta \in$ $\left(0, K_{6}\right)$, the problem $\left(P_{\lambda, \mu, \zeta}\right)$ has at least one positive solution $(u(t), v(t), w(t)), t \in[0,1]$.

Theorem 2.14 Assume that (H1)-(H2) hold. If $g_{\infty}^{i}=h_{\infty}^{i}=\infty, f_{0}^{s}, g_{0}^{s}, h_{0}^{s}, f_{\infty}^{i} \in(0, \infty), a \in$ $[0,1], b \in(0,1), c \in[0,1], d \in(0,1), K_{1}<K_{2}$, then for $\lambda \in\left(K_{1}, K_{2}\right), \mu \in\left(0, K_{4}\right)$, and $\zeta \in$ $\left(0, K_{6}\right)$, the problem $\left(P_{\lambda, \mu, \zeta}\right)$ has at least one positive solution $(u(t), v(t), w(t)), t \in[0,1]$.

Theorem 2.15 Assume that (H1)-(H2) hold. If $f_{\infty}^{i}=g_{\infty}^{i}=h_{\infty}^{i}=\infty, f_{0}^{s}, g_{0}^{s}, h_{0}^{s} \in(0, \infty), a \in$ $[0,1], b \in(0,1), c \in[0,1], d \in(0,1)$, then for $\lambda \in\left(0, K_{2}\right), \mu \in\left(0, K_{4}\right)$, and $\zeta \in\left(0, K_{6}\right)$, the problem $\left(P_{\lambda, \mu, \zeta}\right)$ has at least one positive solution $(u(t), v(t), w(t)), t \in[0,1]$.

Denote the positive constants

$$
\begin{array}{ll}
L_{1}=\frac{b}{\gamma \tilde{A} f_{0}^{i}}, & L_{2}=\frac{c}{A f_{\infty}^{s}}, \quad L_{3}=\frac{a}{\gamma \tilde{B} g_{0}^{i}}, \\
L_{4}=\frac{d}{B g_{\infty}^{s}}, & L_{5}=\frac{1-a-b}{\gamma \tilde{C} h_{0}^{i}}, \quad L_{6}=\frac{1-c-d}{C h_{\infty}^{s}} .
\end{array}
$$

Theorem 2.16 Assume that (H1)-(H2) hold, $f_{0}^{i}, g_{0}^{i}, h_{0}^{i}, f_{\infty}^{s}, g_{\infty}^{s}, h_{\infty}^{s} \in(0, \infty), a \in[0,1], b \in$ $(0,1), c \in[0,1], d \in(0,1), L_{1}<L_{2}, L_{3}<L_{4}$, and $L_{5}<L_{6}$. Then for $\lambda \in\left(L_{1}, L_{2}\right), \mu \in\left(L_{3}, L_{4}\right), \zeta \in$ $\left(L_{5}, L_{6}\right)$, the problem $\left(P_{\lambda, \mu, \zeta}\right)$ has at least one positive solution $(u(t), v(t), w(t)), t \in[0,1]$. 
Proof Let $\lambda \in\left(L_{1}, L_{2}\right), \mu \in\left(L_{3}, L_{4}\right), \zeta \in\left(L_{5}, L_{6}\right), \varepsilon>0$ satisfying $f_{0}^{i}>\varepsilon, g_{0}^{i}>\varepsilon, h_{0}^{i}>\varepsilon$, and

$$
\begin{aligned}
& \frac{b}{\gamma \tilde{A}\left(f_{0}^{i}-\varepsilon\right)}<\lambda<\frac{c}{A\left(f_{\infty}^{s}+\varepsilon\right)}, \\
& \frac{a}{\gamma \tilde{B}\left(g_{0}^{i}-\varepsilon\right)}<\mu<\frac{d}{B\left(g_{\infty}^{s}+\varepsilon\right)}, \\
& \frac{1-a-b}{\gamma \tilde{C}\left(h_{0}^{i}-\varepsilon\right)}<\zeta<\frac{1-c-d}{C\left(h_{\infty}^{s}+\varepsilon\right)} .
\end{aligned}
$$

By condition (H1), there exists $R_{3}>0$ such that for $t \in[\xi, \eta], u(t), v(t), w(t) \geq 0$ and $u(t)+$ $v(t)+w(t) \leq R_{3}$

$$
\begin{aligned}
& f(t, u(t), v(t), w(t)) \geq\left(f_{0}^{i}-\varepsilon\right)(u(t)+v(t)+w(t)), \\
& g(t, u(t), v(t), w(t)) \geq\left(g_{0}^{i}-\varepsilon\right)(u(t)+v(t)+w(t)), \\
& h(t, u(t), v(t), w(t)) \geq\left(h_{0}^{i}-\varepsilon\right)(u(t)+v(t)+w(t)) .
\end{aligned}
$$

We define the set

$$
\Omega_{3}=\left\{(u(t), v(t), w(t)) \in Y,\|(u, v, w)\|_{Y}<R_{3}\right\} .
$$

Let $(u, v, w) \in P \cap \partial \Omega_{3}$,

$$
\begin{aligned}
& T_{1}(u, v, w)(t)=\lambda \int_{0}^{1} k_{1}(t, s) f(s, u(s), v(s), w(s)) d s \\
& \geq \lambda \int_{\xi}^{\eta} k_{1}(t, s) f(s, u(s), v(s), w(s)) d s \\
& \geq \lambda \int_{\xi}^{\eta} k_{1}(t, s)\left(f_{0}^{i}-\varepsilon\right)(u(s)+v(s)+w(s)) d s \\
& \geq \lambda \gamma \int_{\xi}^{\eta} k_{1}(t, s)\left(f_{0}^{i}-\varepsilon\right)\|(u, v, w)\|_{Y} d s \\
& \geq \lambda \gamma \tilde{A}\left(f_{0}^{i}-\varepsilon\right)\|(u, v, w)\|_{Y} \\
& \geq b\|(u, v, w)\|_{Y^{\prime}} \\
& T_{2}(u, v, w)(t)=\mu \int_{0}^{1} k_{2}(t, s) g(s, u(s), v(s), w(s)) d s \\
& \geq \mu \int_{\xi}^{\eta} k_{2}(t, s) g(s, u(s), v(s), w(s)) d s \\
& \geq \mu \int_{\xi}^{\eta} k_{2}(t, s)\left(g_{0}^{i}-\varepsilon\right)(u(s)+v(s)+w(s)) d s \\
& \geq \mu \gamma \int_{\xi}^{\eta} k_{2}(t, s)\left(g_{0}^{i}-\varepsilon\right)\|(u, v, w)\|_{Y} d s \\
& \geq \mu \gamma \tilde{B}\left(g_{0}^{i}-\varepsilon\right)\|(u, v, w)\|_{Y} \\
& \geq a\|(u, v, w)\|_{Y},
\end{aligned}
$$




$$
\begin{aligned}
T_{3}(u, v, w)(t) & =\zeta \int_{0}^{1} k_{3}(t, s) h(s, u(s), v(s)) d s \\
& \geq \zeta \int_{\xi}^{\eta} k_{3}(t, s) h(s, u(s), v(s), w(s)) d s \\
& \geq \zeta \int_{\xi}^{\eta} k_{3}(t, s)\left(h_{0}^{i}-\varepsilon\right)(u(s)+v(s)+w(s)) d s \\
& \geq \zeta \gamma \int_{\xi}^{\eta} k_{3}(t, s)\left(h_{0}^{i}-\varepsilon\right)\|(u, v, w)\|_{Y} d s \\
& \geq \zeta \gamma \tilde{C}\left(h_{0}^{i}-\varepsilon\right)\|(u, v, w)\|_{Y} \\
& \geq(1-a-b)\|(u, v, w)\|_{Y} .
\end{aligned}
$$

Then for $(u, v, w) \in P \cap \partial \Omega_{3}$,

$$
\begin{aligned}
\|T(u, v, w)\|_{Y} & =\left\|T_{1}(u, v, w)\right\|+\left\|T_{2}(u, v, w)\right\|+\left\|T_{3}(u, v, w)\right\| \\
& \geq b\|(u, v, w)\|_{Y}+a\|(u, v, w)\|_{Y}+(1-a-b)\|(u, v, w)\|_{Y} \\
& =\|(u, v, w)\|_{Y} .
\end{aligned}
$$

On the other side, we define the functions $f^{*}, g^{*}, h^{*}[0,1] \times R^{+} \longrightarrow R^{+}$,

$$
\begin{array}{ll}
f^{*}(t, x)=\max _{0 \leq u+v+w \leq x} f(t, u, v, w), & t \in[0,1], x \geq 0, \\
g^{*}(t, x)=\max _{0 \leq u+v+w \leq x} g(t, u, v, w), & t \in[0,1], x \geq 0 . \\
h^{*}(t, x)=\max _{0 \leq u+v+w \leq x} h(t, u, v, w), & t \in[0,1], x \geq 0 .
\end{array}
$$

Then

$$
\begin{array}{ll}
f(t, u, v, w) \leq f^{*}(t, x), & t \in[0,1], u, v, w \geq 0, u+v+w \leq x \\
g(t, u, v, w) \leq g^{*}(t, x), & t \in[0,1], u, v, w \geq 0, u+v+w \leq x \\
h(t, u, v, w) \leq h^{*}(t, x), & t \in[0,1], u, v, w \geq 0, u+v+w \leq x .
\end{array}
$$

The functions $f^{*}(t, \cdot), g^{*}(t, \cdot), h^{*}(t, \cdot)$ are nondecreasing for each $t \in[0,1]$ and satisfy the conditions

$$
\begin{aligned}
& \limsup _{x \rightarrow \infty} \max _{t \in[0,1]} \frac{f^{*}(t, x)}{x} \leq f_{\infty}^{s}, \quad \limsup _{x \rightarrow \infty} \max _{t \in[0,1]} \frac{g^{*}(t, x)}{x} \leq g_{\infty}^{s}, \\
& \limsup _{x \rightarrow \infty} \max _{t \in[0,1]} \frac{h^{*}(t, x)}{x} \leq h_{\infty}^{s},
\end{aligned}
$$

which can be proved similar to Lemma 2.8 in [56]. Thus, for $\varepsilon>0$, there exists $\bar{R}_{4}>0$ such that for all $x \geq \bar{R}_{4}, t \in[0,1]$,

$$
\begin{aligned}
& \frac{f^{*}(t, x)}{x} \leq \limsup _{x \rightarrow \infty} \max _{t \in[0,1]} \frac{f^{*}(t, x)}{x}+\varepsilon \leq f_{\infty}^{s}+\varepsilon, \\
& \frac{g^{*}(t, x)}{x} \leq \limsup _{x \rightarrow \infty} \max _{t \in[0,1]} \frac{g^{*}(t, x)}{x}+\varepsilon \leq g_{\infty}^{s}+\varepsilon,
\end{aligned}
$$




$$
\frac{h^{*}(t, x)}{x} \leq \limsup _{x \rightarrow \infty} \max _{t \in[0,1]} \frac{h^{*}(t, x)}{x}+\varepsilon \leq h_{\infty}^{s}+\varepsilon .
$$

Then

$$
f^{*}(t, x) \leq\left(f_{\infty}^{s}+\varepsilon\right) x, \quad g^{*}(t, x) \leq\left(g_{\infty}^{s}+\varepsilon\right) x, \quad h^{*}(t, x) \leq\left(h_{\infty}^{s}+\varepsilon\right) x
$$

Let $R_{4}=\max \left\{2 R_{3}, \bar{R}_{4}\right\}$ and $\Omega_{4}=\left\{(u, v, w) \in Y,\|(u, v, w)\|_{Y}<R_{4}\right\}$. Let $(u, v, w) \in P \cap \partial \Omega_{4}$, then

$$
\begin{array}{ll}
f(t, u, v, w) \leq f^{*}\left(t,\|(u, v, w)\|_{Y}\right), & t \in[0,1], \\
g(t, u, v, w) \leq g^{*}\left(t,\|(u, v, w)\|_{Y}\right), & t \in[0,1], \\
h(t, u, v, w) \leq h^{*}\left(t,\|(u, v, w)\|_{Y}\right), & t \in[0,1] .
\end{array}
$$

Thus

$$
\begin{aligned}
& T_{1}(u, v, w)(t)=\lambda \int_{0}^{1} k_{1}(t, s) f(s, u(s), v(s), w(s)) d s \\
& \leq \lambda \int_{0}^{1} k_{1}(t, s) f^{*}\left(t,\|(u, v, w)\|_{Y}\right) d s \\
& \leq \lambda \int_{0}^{1} k_{1}(t, s)\left(f_{\infty}^{s}+\varepsilon\right)\|(u, v, w)\|_{Y} d s \\
& \leq \lambda A\left(f_{\infty}^{s}+\varepsilon\right)\|(u, v, w)\|_{Y} \\
& \leq c\|(u, v, w)\|_{Y} \\
& T_{2}(u, v, w)(t)=\mu \int_{0}^{1} k_{2}(t, s) g(s, u(s), v(s), w(s)) d s \\
& \leq \mu \int_{0}^{1} k_{2}(t, s) g^{*}\left(t,\|(u, v, w)\|_{Y}\right) d s \\
& \leq \mu \int_{0}^{1} k_{2}(t, s)\left(g_{\infty}^{s}+\varepsilon\right)\|(u, v, w)\|_{Y} d s \\
& \leq \mu B\left(g_{\infty}^{s}+\varepsilon\right)\|(u, v, w)\|_{Y} \\
& \leq d\|(u, v, w)\|_{Y}, \\
& T_{3}(u, v, w)(t)=\zeta \int_{0}^{1} k_{3}(t, s) h(s, u(s), v(s), w(s)) d s \\
& \leq \zeta \int_{0}^{1} k_{3}(t, s) h^{*}\left(t,\|(u, v, w(s))\|_{Y}\right) d s \\
& \leq \zeta \int_{0}^{1} k_{3}(t, s)\left(h_{\infty}^{s}+\varepsilon\right)\|(u, v, w)\|_{Y} d s \\
& \leq \zeta C\left(h_{\infty}^{s}+\varepsilon\right)\|(u, v, w)\|_{Y} \\
& \leq(1-c-d)\|(u, v, w)\|_{Y} \text {. }
\end{aligned}
$$


Then for $(u, v, w) \in P \cap \partial \Omega_{4}$,

$$
\begin{aligned}
\|T(u, v, w)\|_{Y} & =\left\|T_{1}(u, v, w)\right\|+\left\|T_{2}(u, v, w)\right\|+\left\|T_{3}(u, v, w)\right\| \\
& \leq c\|(u, v, w)\|_{Y}+d\|(u, v, w)\|_{Y}+(1-c-d)\|(u, v, w)\|_{Y} \\
& =\|(u, v, w)\|_{Y} .
\end{aligned}
$$

By using Lemma 1.1, $T$ has a fixed point $(u, v, w) \in P \cap\left(\Omega_{4} \backslash \bar{\Omega}_{3}\right)$.

We can also consider the case that the above limits reach 0 or $\infty$.

Theorem 2.17 Assume that (H1)-(H2) hold. If $f_{\infty}^{s}=0, f_{0}^{i}, g_{0}^{i}, h_{0}^{i}, g_{\infty}^{s}, h_{\infty}^{s} \in(0, \infty), a \in$ $[0,1], b \in(0,1), c \in[0,1], d \in(0,1), L_{3}<L_{4}$, and $L_{5}<L_{6}$, then for $\lambda \in\left(L_{1}, \infty\right), \mu \in\left(L_{3}, L_{4}\right)$, $\zeta \in\left(L_{5}, L_{6}\right)$, the problem $\left(P_{\lambda, \mu, \zeta}\right)$ has at least one positive solution $(u(t), v(t), w(t)), t \in[0,1]$.

Theorem 2.18 Assume that (H1)-(H2) hold. If $g_{\infty}^{s}=0, f_{0}^{i}, g_{0}^{i}, h_{0}^{i}, f_{\infty}^{s}, h_{\infty}^{s} \in(0, \infty), a \in$ $[0,1], b \in(0,1), c \in[0,1], d \in(0,1), L_{1}<L_{2}$, and $L_{5}<L_{6}$, then for $\lambda \in\left(L_{1}, L_{2}\right), \mu \in\left(L_{3}, \infty\right)$, $\zeta \in\left(L_{5}, L_{6}\right)$, the problem $\left(P_{\lambda, \mu, \zeta}\right)$ has at least one positive solution $(u(t), v(t), w(t)), t \in[0,1]$.

Theorem 2.19 Assume that (H1)-(H2) hold. If $h_{\infty}^{s}=0, f_{0}^{i}, g_{0}^{i}, h_{0}^{i}, g_{\infty}^{s}, f_{\infty}^{s} \in(0, \infty), a \in$ $[0,1], b \in(0,1), c \in[0,1], d \in(0,1), L_{1}<L_{2}$, and $L_{3}<L_{4}$, then for $\lambda \in\left(L_{2}, L_{2}\right), \mu \in\left(L_{3}, L_{4}\right)$, $\zeta \in\left(L_{5}, \infty\right)$, the problem $\left(P_{\lambda, \mu, \zeta}\right)$ has at least one positive solution $(u(t), v(t), w(t)), t \in[0,1]$.

Theorem 2.20 Assume that (H1)-(H2) hold. If $f_{\infty}^{s}=g_{\infty}^{s}=0, f_{0}^{i}, g_{0}^{i}, h_{0}^{i}, h_{\infty}^{s} \in(0, \infty), a \in$ $[0,1], b \in(0,1), c \in[0,1], d \in(0,1)$, and $L_{5}<L_{6}$, then for $\lambda \in\left(L_{1}, \infty\right), \mu \in\left(L_{3}, \infty\right), \zeta \in$ $\left(L_{5}, L_{6}\right)$, the problem $\left(P_{\lambda, \mu, \zeta}\right)$ has at least one positive solution $(u(t), v(t), w(t)), t \in[0,1]$.

Theorem 2.21 Assume that (H1)-(H2) hold. If $f_{\infty}^{s}=h_{\infty}^{s}=0, f_{0}^{i}, g_{0}^{i}, h_{0}^{i}, g_{\infty}^{s} \in(0, \infty), a \in$ $[0,1], b \in(0,1), c \in[0,1], d \in(0,1)$, and $L_{3}<L_{4}$, then for $\lambda \in\left(L_{1}, \infty\right), \mu \in\left(L_{3}, L_{4}\right), \zeta \in$ $\left(L_{5}, \infty\right)$, the problem $\left(P_{\lambda, \mu, \zeta}\right)$ has at least one positive solution $(u(t), v(t), w(t)), t \in[0,1]$.

Theorem 2.22 Assume that (H1)-(H2) hold. If $h_{\infty}^{s}=g_{\infty}^{s}=0, f_{0}^{i}, g_{0}^{i}, h_{0}^{i}, f_{\infty}^{s} \in(0, \infty), a \in$ $[0,1], b \in(0,1), c \in[0,1], d \in(0,1)$, and $L_{1}<L_{2}$, then for $\lambda \in\left(L_{1}, L_{2}\right), \mu \in\left(L_{3}, \infty\right), \zeta \in$ $\left(L_{5}, \infty\right)$, the problem $\left(P_{\lambda, \mu, \zeta}\right)$ has at least one positive solution $(u(t), v(t), w(t)), t \in[0,1]$.

Theorem 2.23 Assume that (H1)-(H2) hold. If $f_{\infty}^{s}=g_{\infty}^{s}=h_{\infty}^{s}=0, f_{0}^{i}, g_{0}^{i}, h_{0}^{i} \in(0, \infty), a \in$ $[0,1], b \in(0,1), c \in[0,1], d \in(0,1)$, then for $\lambda \in\left(L_{1}, \infty\right), \mu \in\left(L_{3}, \infty\right), \zeta \in\left(L_{5}, \infty\right)$, the problem $\left(P_{\lambda, \mu, \zeta}\right)$ has at least one positive solution $(u(t), v(t), w(t)), t \in[0,1]$.

Theorem 2.24 Assume that (H1)-(H2) hold. If $f_{0}^{i}=\infty, g_{0}^{i}, h_{0}^{i}, f_{\infty}^{s}, g_{\infty}^{s}, h_{\infty}^{s} \in(0, \infty), a \in$ $[0,1], b \in(0,1), c \in[0,1], d \in(0,1), L_{3}<L_{4}$, and $L_{5}<L_{6}$, then for $\lambda \in\left(0, L_{2}\right), \mu \in\left(L_{3}, L_{4}\right), \zeta \in$ $\left(L_{5}, L_{6}\right)$, the problem $\left(P_{\lambda, \mu, \zeta}\right)$ has at least one positive solution $(u(t), v(t), w(t)), t \in[0,1]$.

Theorem 2.25 Assume that (H1)-(H2) hold. If $g_{0}^{i}=\infty, f_{0}^{i}, h_{0}^{i}, f_{\infty}^{s}, g_{\infty}^{s}, h_{\infty}^{s} \in(0, \infty), a \in$ $[0,1], b \in(0,1), c \in[0,1], d \in(0,1), L_{1}<L_{2}$, and $L_{5}<L_{6}$, then for $\lambda \in\left(L_{1}, L_{2}\right), \mu \in\left(0, L_{4}\right), \zeta \in$ $\left(L_{5}, L_{6}\right)$, the problem $\left(P_{\lambda, \mu, \zeta}\right)$ has at least one positive solution $(u(t), v(t), w(t)), t \in[0,1]$. 
Theorem 2.26 Assume that (H1)-(H2) hold. If $h_{0}^{i}=\infty, g_{0}^{i}, f_{0}^{i}, f_{\infty}^{s}, g_{\infty}^{s}, h_{\infty}^{s} \in(0, \infty), a \in$ $[0,1], b \in(0,1), c \in[0,1], d \in(0,1), L_{3}<L_{4}$, and $L_{1}<L_{2}$, then for $\lambda \in\left(L_{1}, L_{2}\right), \mu \in\left(L_{3}, L_{4}\right)$, $\zeta \in\left(0, L_{6}\right)$, the problem $\left(P_{\lambda, \mu, \zeta}\right)$ has at least one positive solution $(u(t), v(t), w(t)), t \in[0,1]$.

Theorem 2.27 Assume that (H1)-(H2) hold. If $f_{0}^{i}=g_{0}^{i}=\infty, h_{0}^{i}, f_{\infty}^{s}, g_{\infty}^{s}, h_{\infty}^{s} \in(0, \infty), a \in$ $[0,1], b \in(0,1), c \in[0,1], d \in(0,1)$, and $L_{5}<L_{6}$, then for $\lambda \in\left(0, L_{2}\right), \mu \in\left(0, L_{4}\right), \zeta \in\left(L_{5}, L_{6}\right)$, the problem $\left(P_{\lambda, \mu, \zeta}\right)$ has at least one positive solution $(u(t), v(t), w(t)), t \in[0,1]$.

Theorem 2.28 Assume that (H1)-(H2) hold. If $f_{0}^{i}=h_{0}^{i}=\infty, g_{0}^{i}, f_{\infty}^{s}, g_{\infty}^{s}, h_{\infty}^{s} \in(0, \infty), a \in$ $[0,1], b \in(0,1), c \in[0,1], d \in(0,1)$, and $L_{3}<L_{4}$, then for $\lambda \in\left(0, L_{2}\right), \mu \in\left(L_{3}, L_{4}\right), \zeta \in\left(0, L_{6}\right)$, the problem $\left(P_{\lambda, \mu, \zeta}\right)$ has at least one positive solution $(u(t), v(t), w(t)), t \in[0,1]$.

Theorem 2.29 Assume that (H1)-(H2) hold. If $h_{0}^{i}=g_{0}^{i}=\infty, f_{0}^{i}, f_{\infty}^{s}, g_{\infty}^{s}, h_{\infty}^{s} \in(0, \infty), a \in$ $[0,1], b \in(0,1), c \in[0,1], d \in(0,1)$, and $L_{1}<L_{2}$, then for $\lambda \in\left(L_{1}, L_{2}\right), \mu \in\left(0, L_{4}\right), \zeta \in\left(0, L_{6}\right)$, the problem $\left(P_{\lambda, \mu, \zeta}\right)$ has at least one positive solution $(u(t), v(t), w(t)), t \in[0,1]$.

Theorem 2.30 Assume that (H1)-(H2) hold. If $f_{0}^{i}=g_{0}^{i}=h_{0}^{i}=\infty, f_{\infty}^{s}, g_{\infty}^{s}, h_{\infty}^{s} \in(0, \infty), a \in$ $[0,1], b \in(0,1), c \in[0,1], d \in(0,1)$, then for $\lambda \in\left(0, L_{2}\right), \mu \in\left(0, L_{4}\right), \zeta \in\left(0, L_{6}\right)$, the problem $\left(P_{\lambda, \mu, \zeta}\right)$ has at least one positive solution $(u(t), v(t), w(t)), t \in[0,1]$.

The proof is similar to Theorem 2.16, we omit it here.

\section{Nonexistence results of positive solutions}

In this section we shall consider sufficient conditions on $\lambda, \mu, \zeta, f, g$, and $h$ such that the problem $\left(P_{\lambda, \mu, \zeta}\right)$ has no positive solution.

Theorem 3.1 Assume that (H1)-(H2) hold. If $f_{0}^{s}, f_{\infty}^{s}, g_{0}^{s}, g_{\infty}^{s}, h_{0}^{s}, h_{\infty}^{s}<\infty$, then there exists a positive constant $\lambda_{0}, \mu_{0}, \zeta_{0}$ such that for every $\lambda \in\left(0, \lambda_{0}\right), \mu \in\left(0, \mu_{0}\right), \zeta \in\left(0, \zeta_{0}\right)$, the problem $\left(P_{\lambda, \mu, \zeta}\right)$ has no positive solution.

Proof From the condition $f_{0}^{s}, f_{\infty}^{s}, g_{0}^{s}, g_{\infty}^{s}, h_{0}^{s}, h_{\infty}^{s}<\infty$, there exist $M_{1}>0, M_{2}>0, M_{3}>0$ such that

$$
\begin{aligned}
& f(t, u, v, w) \leq M_{1}(u+v+w), \quad g(t, u, v, w) \leq M_{2}(u+v+w), \\
& h(t, u, v, w) \leq M_{3}(u+v+w), \quad t \in[0,1], u, v, w \geq 0 .
\end{aligned}
$$

Define the positive constants

$$
\lambda_{0}=\frac{a}{A M_{1}}, \quad \mu_{0}=\frac{b}{B M_{2}}, \quad \zeta_{0}=\frac{1-a-b}{C M_{3}} .
$$

Let $\lambda \in\left(0, \lambda_{0}\right), \mu \in\left(0, \mu_{0}\right), \zeta \in\left(0, \zeta_{0}\right)$, suppose that the problem $\left(P_{\lambda, \mu, \zeta}\right)$ has a positive solution $(u(t), v(t)), t \in[0,1]$. Thus,

$$
\begin{aligned}
T_{1}(u, v, w)(t) & =\lambda \int_{0}^{1} k_{1}(t, s) f(s, u(s), v(s), w(s)) d s \\
& \leq \lambda \int_{0}^{1} k_{1}(t, s) M_{1}(u(s)+v(s)+w(s)) d s
\end{aligned}
$$




$$
\begin{aligned}
& \leq \lambda M_{1} \int_{0}^{1} k_{1}(t, s)(\|u\|+\|v\|+\|w\|) d s \\
& \leq \lambda A M_{1}(\|u\|+\|v\|+\|w\|) \\
& <\lambda_{0} A M_{1}\|(u, v, w)\|_{Y} \\
& <a\|(u, v, w)\|_{Y}, \\
T_{2}(u, v, w)(t) & =\mu \int_{0}^{1} k_{2}(t, s) g(s, u(s), v(s), w(s)) d s \\
& \leq \mu \int_{0}^{1} k_{2}(t, s) M_{2}(u(s)+v(s)+w(s)) d s \\
& \leq \mu M_{2} \int_{0}^{1} k_{2}(t, s)(\|u\|+\|v\|+\|w\|) d s \\
& \leq \mu B M_{2}(\|u\|+\|v\|+\|w\|) \\
& <\mu_{0} B M_{2}\|(u, v, w)\|_{Y}, \\
& <b\|(u, v, w)\|_{Y}, \\
& \leq \zeta M_{3} \int_{0}^{1} k_{3}(t, s)(\|u\|+\|v\|+\|w\|) d s \\
& \leq \zeta C M_{3}(\|u\|+\|v\|+\|w\|) \\
& \leq \zeta \int_{0}^{1} k_{3}(t, s) M_{3}(u(s)+v(s)+w(s)) d s \\
T_{3}(u, v, w)(t) & =\zeta \int_{0}(t, s) h(s, u(s), v(s), w(s)) d s \\
& \\
& \\
& \\
& \\
&
\end{aligned}
$$

Then

$$
\begin{aligned}
\|(u, v, w)\|_{Y} & =\left\|T_{1}(u, v, w)\right\|+\left\|T_{2}(u, v, w)\right\|+\left\|T_{3}(u, v, w)\right\| \\
& <a\|(u, v, w)\|_{Y}+b\|(u, v, w)\|_{Y}+(1-a-b)\|(u, v, w)\|_{Y} \\
& =\|(u, v, w)\|_{Y}
\end{aligned}
$$

which is a contradiction. So the boundary value problem $\left(P_{\lambda, \mu, \zeta}\right)$ has no positive solution.

Theorem 3.2 Assume that $(\mathrm{H} 1)-(\mathrm{H} 2)$ hold.If $f_{0}^{i}, f_{\infty}^{i}>0$, then there exists a positive constant $\tilde{\lambda}_{0}$ such that for every $\lambda \in\left(\tilde{\lambda}_{0}, \infty\right), \mu>0, \zeta>0$, the boundary value problem $\left(P_{\lambda, \mu, \zeta}\right)$ has no positive solution.

Proof From the definitions of $f_{0}^{i}, f_{\infty}^{i}$, and the condition $f_{0}^{i}, f_{\infty}^{i}>0$, there exist positive numbers $m_{1}$ such that

$$
f(t, u, v, w) \geq m_{1}(u+v+w), \quad t \in[\xi, \eta], u, v, w \geq 0
$$


Define thepositive constants

$$
\tilde{\lambda}_{0}=\frac{1}{\gamma \tilde{A} m_{1}} .
$$

Let $\lambda \in\left(\tilde{\lambda}_{0}, \infty\right), \mu>0, \zeta>0$, we suppose that the problem $\left(P_{\lambda, \mu, \zeta}\right)$ has a positive solution $(u(t), v(t), w(t)), t \in[0,1]$. Then for $t \in[0,1]$, we have

$$
\begin{aligned}
T_{1}(u, v, w)(t) & =\lambda \int_{0}^{1} k_{1}(t, s) f(s, u(s), v(s), w(s)) d s \\
& \geq \lambda \int_{\xi}^{\eta} k_{1}(t, s) f(s, u(s), v(s), w(s)) d s \\
& \geq \lambda \int_{\xi}^{\eta} k_{1}(t, s) m_{1}(u(s)+v(s)+w(s)) d s \\
& \geq \lambda \gamma \int_{\xi}^{\eta} k_{1}(t, s) m_{1}\|(u, v, w)\|_{Y} d s \\
& \geq \lambda \gamma \tilde{A} m_{1}\|(u, v, w)\|_{Y} \\
& >\|(u, v, w)\|_{Y} .
\end{aligned}
$$

Thus,

$$
\|(u, v, w)\|_{Y} \geq\left\|T_{1}(u, v, w)\right\|>\|(u, v, w)\|_{Y},
$$

which is a contradiction. So the boundary value problem $\left(P_{\lambda, \mu, \zeta}\right)$ has no positive solution.

Theorem 3.3 Assume that (H1)-( $\mathrm{H} 2)$ hold. If $g_{0}^{i}, g_{\infty}^{i}>0$, then there exists a positive constant $\tilde{\mu}_{0}$ such that for every $\mu \in\left(\tilde{\mu}_{0}, \infty\right), \lambda>0, \zeta>0$, the boundary value problem $\left(P_{\lambda, \mu, \zeta}\right)$ has no positive solution.

Theorem 3.4 Assume that $(\mathrm{H} 1)-(\mathrm{H} 2)$ hold. If $h_{0}^{i}, h_{\infty}^{i}>0$, then there exists a positive constant $\tilde{\zeta}_{0}$ such that for every $\zeta \in\left(\tilde{\zeta}_{0}, \infty\right), \lambda>0, \mu>0$, the boundary value problem $\left(P_{\lambda, \mu, \zeta}\right)$ has no positive solution.

Theorem 3.5 Assume that (H1)-(H2) hold. If $f_{0}^{i}, f_{\infty}^{i}, g_{0}^{i}, g_{\infty}^{i}>0$, then there exist positive constants $\tilde{\lambda}_{0}, \tilde{\mu}_{0}, a \in[0,1]$, such that for every $\lambda \in\left(\tilde{\lambda}_{0}, \infty\right), \mu \in\left(\tilde{\mu}_{0}, \infty\right), \zeta>0$, the boundary value problem $\left(P_{\lambda, \mu, \zeta}\right)$ has no positive solution.

Proof From the definitions of $f_{0}^{i}, f_{\infty}^{i}, g_{0}^{i}, g_{\infty}^{i}$, and the condition $f_{0}^{i}, f_{\infty}^{i}, g_{0}^{i}, g_{\infty}^{i}>0$, there exist positive numbers $m_{1}, m_{2}$ such that

$$
\begin{array}{ll}
f(t, u, v, w) \geq m_{1}(u+v+w), & t \in[\xi, \eta], u, v, w \geq 0 . \\
g(t, u, v, w) \geq m_{2}(u+v+w), & t \in[\xi, \eta], u, v, w \geq 0 .
\end{array}
$$

Define the positive constants

$$
\tilde{\lambda}_{0}=\frac{a}{\gamma \tilde{A} m_{1}}, \quad \tilde{\mu}_{0}=\frac{1-a}{\gamma \tilde{B} m_{2}}
$$


Let $\lambda \in\left(\tilde{\lambda}_{0}, \infty\right), \mu \in\left(\tilde{\mu}_{0}, \infty\right), \zeta>0$, we suppose that the problem $\left(P_{\lambda, \mu, \zeta}\right)$ has a positive solution $(u(t), v(t), w(t)), t \in[0,1]$. Then for $t \in[0,1]$, we have

$$
\begin{aligned}
T_{1}(u, v, w)(t) & =\lambda \int_{0}^{1} k_{1}(t, s) f(s, u(s), v(s), w(s)) d s \\
& \geq \lambda \int_{\xi}^{\eta} k_{1}(t, s) f(s, u(s), v(s), w(s)) d s \\
& \geq \lambda \int_{\xi}^{\eta} k_{1}(t, s) m_{1}(u(s)+v(s)+w(s)) d s \\
& \geq \lambda \gamma \int_{\xi}^{\eta} k_{1}(t, s) m_{1}\|(u, v, w)\|_{Y} d s \\
& \geq \lambda \gamma \tilde{A} m_{1}\|(u, v, w)\|_{Y} \\
& \geq a\|(u, v, w)\|_{Y}, \\
T_{2}(u, v, w)(t) & =\mu \int_{0}^{1} k_{2}(t, s) g(s, u(s), v(s), w(s)) d s \\
& \geq \mu \int_{\xi}^{\eta} k_{2}(t, s) g(s, u(s), v(s), w(s)) d s \\
& \geq \mu \int_{\xi}^{\eta} k_{2}(t, s) m_{2}(u(s)+v(s)+w(s)) d s \\
& \geq \mu \gamma \int_{\xi}^{\eta} k_{2}(t, s) m_{2}\|(u, v, w)\|_{Y} d s \\
& \geq \mu \gamma \tilde{B} m_{2}\|(u, v, w)\|_{Y} \\
& \geq(1-a)\|(u, v, w)\|_{Y} .
\end{aligned}
$$

Thus,

$$
\|(u, v, w)\|_{Y}>a\|(u, v, w)\|_{Y}+(1-a)\|(u, v, w)\|_{Y}=\|(u, v, w)\|_{Y},
$$

which is a contradiction. So the boundary value problem $\left(P_{\lambda, \mu, \zeta}\right)$ has no positive solution.

Theorem 3.6 Assume that (H1)-(H2) hold. If $f_{0}^{i}, f_{\infty}^{i}, h_{0}^{i}, h_{\infty}^{i}>0$, then there exist positive constants $\tilde{\lambda}_{0}, \tilde{\zeta}_{0}$ such that for every $\lambda \in\left(\tilde{\lambda}_{0}, \infty\right), \zeta \in\left(\tilde{\zeta}_{0}, \infty\right), \mu>0$, the boundary value problem $\left(P_{\lambda, \mu, \zeta}\right)$ has no positive solution.

Theorem 3.7 Assume that (H1)-(H2) hold. If $g_{0}^{i}, g_{\infty}^{i}, h_{0}^{i}, h_{\infty}^{i}>0$, then there exist positive constants $\tilde{\mu}_{0}, \tilde{\zeta}_{0}$ such that for every $\mu \in\left(\tilde{\mu}_{0}, \infty\right), \zeta \in\left(\tilde{\zeta}_{0}, \infty\right), \lambda>0$, the boundary value problem $\left(P_{\lambda, \mu, \zeta}\right)$ has no positive solution.

Theorem 3.8 Assume that (H1)-(H2) hold. If $f_{0}^{i}, f_{\infty}^{i}, g_{0}^{i}, g_{\infty}^{i}, h_{0}^{i}, h_{\infty}^{i}>0$, then there exist positive constants $\tilde{\lambda}_{0}, \tilde{\mu}_{0}, \tilde{\zeta}_{0}$ such that for every $\lambda \in\left(\tilde{\lambda}_{0}, \infty\right), \mu \in\left(\tilde{\mu}_{0}, \infty\right), \zeta \in\left(\tilde{\zeta}_{0}, \infty\right)$, the boundary value problem $\left(P_{\lambda, \mu, \zeta}\right)$ has no positive solution. 
Proof From the definitions of $f_{0}^{i}, f_{\infty}^{i}, g_{0}^{i}, g_{\infty}^{i}, h_{0}^{i}, h_{\infty}^{i}$, and the condition

$$
f_{0}^{i}, f_{\infty}^{i}, g_{0}^{i}, g_{\infty}^{i}, h_{0}^{i}, h_{\infty}^{i}>0
$$

there exist positive numbers $m_{1}, m_{2}, m_{3}$ such that

$$
\begin{array}{ll}
f(t, u, v, w) \geq m_{1}(u+v+w), & t \in[0,1], u, v, w \geq 0, \\
g(t, u, v, w) \geq m_{2}(u+v+w), & t \in[0,1], u, v, w \geq 0, \\
h(t, u, v, w) \geq m_{3}(u+v+w), & t \in[0,1], u, v, w \geq 0 .
\end{array}
$$

Define the positive constants

$$
\tilde{\lambda}_{0}=\frac{c}{\gamma \tilde{A} m_{1}}, \quad \tilde{\mu}_{0}=\frac{d}{\gamma \tilde{B} m_{2}}, \quad \tilde{\zeta}_{0}=\frac{1-c-d}{\gamma \tilde{C} m_{3}} .
$$

Let $\lambda \in\left(\tilde{\lambda}_{0}, \infty\right), \mu \in\left(\tilde{\mu}_{0}, \infty\right), \zeta \in\left(\tilde{\zeta}_{0}, \infty\right)$, we suppose that the problem $\left(P_{\lambda, \mu, \zeta}\right)$ has a positive solution $(u(t), v(t), w(t)), t \in[0,1]$. Then for $t \in[0,1]$, we have

$$
\begin{aligned}
T_{1}(u, v, w)(t) & =\lambda \int_{0}^{1} k_{1}(t, s) f(s, u(s), v(s), w(s)) d s \\
& \geq \lambda \int_{\xi}^{\eta} k_{1}(t, s) f(s, u(s), v(s), w(s)) d s \\
& \geq \lambda \int_{\xi}^{\eta} k_{1}(t, s) m_{1}(u(s)+v(s)+w(s)) d s \\
& \geq \lambda \gamma \int_{\xi}^{\eta} k_{1}(t, s) m_{1}\|(u, v, w)\|_{Y} d s \\
& \geq \lambda \gamma \tilde{A} m_{1}\|(u, v, w)\|_{Y} \\
& \geq c\|(u, v, w)\|_{Y}, \\
T_{2}(u, v, w)(t) & =\mu \int_{0}^{1} k_{2}(t, s) g(s, u(s), v(s), w(s)) d s \\
& \geq \mu \int_{\xi}^{\eta} k_{2}(t, s) g(s, u(s), v(s), w(s)) d s \\
& \geq \mu \int_{\xi}^{\eta} k_{2}(t, s) m_{2}(u(s)+v(s)+w(s)) d s \\
& \geq \mu \gamma \int_{\xi}^{\eta} k_{2}(t, s) m_{2}\|(u, v, w)\|_{Y} d s \\
& \geq \mu \gamma \tilde{B} m_{2}\|(u, v, w)\|_{Y} \\
& \geq d\|(u, v, w)\|_{Y}, \\
& \geq \zeta \int_{\xi}^{\eta} k_{3}(t, s) h(s, u(s), v(s), w(s)) d s \\
T_{3}(u, v, w)(t) & =\zeta \int_{0}^{1} k_{3}(t, s) h(s, u(s), v(s), w(s)) d s \\
&
\end{aligned}
$$




$$
\begin{aligned}
& \geq \zeta \int_{\xi}^{\eta} k_{3}(t, s) m_{3}(u(s)+v(s)+w(s)) d s \\
& \geq \zeta \gamma \int_{\xi}^{\eta} k_{3}(t, s) m_{3}\|(u, v, w)\|_{Y} d s \\
& \geq \zeta \gamma \tilde{C} m_{3}\|(u, v, w)\|_{Y} \\
& \geq(1-c-d)\|(u, v, w)\|_{Y} .
\end{aligned}
$$

Thus,

$$
\begin{aligned}
\|(u, v, w)\|_{Y} & =\left\|T_{1}(u, v, w)\right\|+\left\|T_{2}(u, v, w)\right\|+\left\|T_{3}(u, v, w)\right\| \\
& >c\|(u, v, w)\|_{Y}+d\|(u, v, w)\|_{Y}+(1-c-d)\|(u, v, w)\|_{Y} \\
& =\|(u, v, w)\|_{Y},
\end{aligned}
$$

which is a contradiction. So the boundary value problem $\left(P_{\lambda, \mu, \zeta}\right)$ has no positive solution.

\section{Examples}

In this section we show how our results may be applied to consider the existence and nonexistence of positive solutions for a system of boundary value problems for differential equations of integral or fractional order. The study of these problems was mainly initiated by Il'in and Moiseev $[57,58]$. Since then positive solutions of boundary value problems have been extensively studied by many researchers in recent years, not only because of their mathematical interest but also because of their wide use in a variety of applications.

\subsection{Application to system of boundary value problems of ordinary differential equations}

Consider the system of nonlinear second order differential equation (the problem (P1))

$$
\begin{cases}u^{\prime \prime}(t)+\lambda f(t, u(t), v(t), w(t))=0, & t \in[0,1] \\ v^{\prime \prime}(t)+\mu g(t, u(t), v(t), w(t))=0, & t \in[0,1], \\ w^{\prime \prime}(t)+\zeta h(t, u(t), v(t), w(t))=0, & t \in[0,1]\end{cases}
$$

subject to the boundary conditions

$$
\begin{array}{ll}
u(0)=0, & u(1)=\beta_{1} u\left(\eta_{1}\right), \\
v^{\prime}(0)=0, & v(1)=\beta_{2} v\left(\eta_{2}\right), \\
w^{\prime}(0)=0, & w(1)=\beta_{3} w\left(\eta_{3}\right),
\end{array}
$$

where $0<\eta_{1}, \eta_{2}, \eta_{3}<1$, and

$$
\begin{aligned}
& f(t, u, v, w)=\frac{\sqrt[3]{t}(70(u+v+w)+1)(3+\sin (v))(u+v+w)}{u+v+w+1} \\
& g(t, u, v, w)=\frac{\sqrt{t+1}(40(u+v+w)+1)(4+\cos (w))(u+v+w)}{u+v+w+1}
\end{aligned}
$$




$$
h(t, u, v, w)=\frac{\sqrt{t+1}(50(u+v+w)+1)(3+\cos (u))(u+v+w)}{u+v+w+1}
$$

By using the Green's functions, we can formulate the problem (P1) as

$$
\begin{cases}u(t)=\lambda \int_{0}^{1} k_{1}(t, s) f(s, u(s), v(s), w(s)) d s, & 0 \leq t \leq 1 \\ v(t)=\mu \int_{0}^{1} k_{2}(t, s) g(s, u(s), v(s), w(s)) d s, & 0 \leq t \leq 1 \\ w(t)=\zeta \int_{0}^{1} k_{3}(t, s) h(s, u(s), v(s), w(s)) d s, & 0 \leq t \leq 1\end{cases}
$$

where

$$
\begin{aligned}
& k_{1}(t, s)= \begin{cases}\frac{t(1-s)}{1-\beta_{1} \eta_{1}}-\frac{\beta_{1}\left(\eta_{1}-s\right) t}{1-\beta_{1} \eta_{1}}-(t-s), & 0 \leq s \leq t \leq 1, s \leq \eta_{1}, \\
\frac{t(1-s)}{1-\beta_{1} \eta_{1}}-\frac{\beta_{1}\left(\eta_{1}-s\right) t}{1-\beta_{1} \eta_{1}}, & 0 \leq t \leq s \leq \eta_{1}, \\
\frac{t(1-s)}{1-\beta_{1} \eta_{1}}, & 0 \leq t \leq s \leq 1, s \geq \eta_{1}, \\
\frac{t(1-s)}{1-\beta_{1} \eta_{1}}-(t-s), & \eta_{1} \leq s \leq t \leq 1,\end{cases} \\
& k_{2}(t, s)= \begin{cases}\frac{1-s}{1-\beta_{2}}-\frac{\beta_{2}\left(\eta_{2}-s\right) t}{1-\beta_{2}}-(t-s), & 0 \leq s \leq t \leq 1, s \leq \eta_{2}, \\
\frac{1-s}{1-\beta_{2}}-\frac{\beta_{2}\left(\eta_{2}-s\right) t}{1-\beta_{2}}, & 0 \leq t \leq s \leq \eta_{2} \\
\frac{t(1-s)}{1-\beta_{2}}, & 0 \leq t \leq s \leq 1, s \geq \eta_{2}, \\
\frac{1-s}{1-\beta_{2}}-(t-s), & \eta_{1} \leq s \leq t \leq 1,\end{cases}
\end{aligned}
$$

and

$$
k_{3}(t, s)= \begin{cases}\frac{1-s}{1-\beta_{3}}-\frac{\beta_{3}\left(\eta_{3}-s\right) t}{1-\beta_{3}}-(t-s), & 0 \leq s \leq t \leq 1, s \leq \eta_{3} \\ \frac{1-s}{1-\beta_{3}}-\frac{\beta_{3}\left(\eta_{3}-s\right) t}{1-\beta_{3}}, & 0 \leq t \leq s \leq \eta_{3} \\ \frac{t(1-s)}{1-\beta_{3}}, & 0 \leq t \leq s \leq 1, s \geq \eta_{3} \\ \frac{1-s}{1-\beta_{3}}-(t-s), & \eta_{3} \leq s \leq t \leq 1\end{cases}
$$

We consider the case $\eta_{1}=\frac{1}{2}, \eta_{2}=\eta_{3}=\frac{1}{3}, \beta_{1}=\frac{1}{3}, \beta_{2}=\beta_{3}=\frac{1}{2}$. After an easy computation, we conclude

$$
\begin{aligned}
& A=\frac{1}{5}, \quad \tilde{A}=\frac{1}{30}, \quad B=\frac{13}{9}, \quad \tilde{B}=\frac{26}{45}, \quad C=\frac{13}{9}, \quad \tilde{C}=\frac{26}{45}, \quad \gamma=\frac{1}{6}, \\
& f_{0}^{s}=3, \quad f_{0}^{i}=3 \sqrt[3]{\frac{1}{2}}, \quad f_{\infty}^{s}=280, \quad f_{\infty}^{i}=140 \sqrt[3]{\frac{1}{2}} \\
& g_{0}^{s}=4 \sqrt{2}, \quad g_{0}^{i}=4, \quad g_{\infty}^{s}=200 \sqrt{2}, \quad g_{\infty}^{i}=120, \\
& h_{0}^{s}=3 \sqrt{2}, \quad h_{0}^{i}=3, \quad h_{\infty}^{s}=200 \sqrt{2}, \quad h_{\infty}^{i}=100, \\
& M_{1}=280, \quad M_{2}=200 \sqrt{2}, \quad M_{3}=200 \sqrt{2}, \\
& m_{1}=3 \sqrt[3]{\frac{1}{2}}, \quad m_{2}=4, \quad m_{3}=3, \quad a=b=c=d=\frac{1}{3},
\end{aligned}
$$

and

$$
\begin{array}{ll}
K_{1}=\frac{b}{\gamma \tilde{A} f_{\infty}^{i}} \approx 0.5400, & K_{2}=\frac{a}{A f_{0}^{s}} \approx 0.5556, \quad K_{3}=\frac{1-b}{\gamma \tilde{B} g_{\infty}^{i}} \approx 0.0288, \\
K_{4}=\frac{1-a}{B g_{0}^{s}} \approx 0.0408, \quad K_{5}=\frac{1-a-b}{\gamma \tilde{C} h_{\infty}^{i}} \approx 0.0346, \quad K_{6}=\frac{1-c-d}{C h_{0}^{s}} \approx 0.0544,
\end{array}
$$




$$
\begin{aligned}
& \lambda_{0}=\frac{a}{A M_{1}} \approx 0.0060, \quad \mu_{0}=\frac{b}{B M_{2}} \approx 0.0008, \quad \zeta_{0}=\frac{1-a-b}{C M_{3}} \approx 0.0008, \\
& \tilde{\lambda}_{0}=\frac{c}{\gamma \tilde{A} m_{1}} \approx 25.1894, \quad \tilde{\mu}_{0}=\frac{d}{\gamma \tilde{B} m_{2}} \approx 0.8654, \quad \tilde{\zeta}_{0}=\frac{1-c-d}{\gamma \tilde{C} m_{3}} \approx 1.1538 .
\end{aligned}
$$

Then:

(1) from Theorem 2.1, for $\lambda \in\left(K_{1}, K_{2}\right), \mu \in\left(K_{3}, K_{4}\right), \zeta \in\left(K_{5}, K_{6}\right)$, the problem (P1) has a positive solution;

(2) from Theorem 3.1, for $\lambda \in\left(0, \lambda_{0}\right), \mu \in\left(0, \mu_{0}\right), \zeta \in\left(0, \zeta_{0}\right)$, the problem (P1) has no positive solution;

(3) from Theorem 3.8, for $\lambda \in\left(\tilde{\lambda}_{0}, \infty\right), \mu \in\left(\tilde{\mu}_{0}, \infty\right), \zeta \in\left(\tilde{\zeta}_{0}, \infty\right)$ the problem (P1) has no positive solution.

\subsection{Application to system of boundary value problems of differential equations on time scales}

Consider the system of boundary value problems of nonlinear differential equation on time scale $\mathbb{T}$ (the problem (P2)),

$$
\begin{array}{ll}
u^{\Delta \nabla}(t)+\lambda f(t, u(t), v(t), w(t))=0, & t \in[0, T] \subset \mathbb{T}, \\
v^{\Delta \nabla}(t)+\mu g(t, u(t), v(t), w(t))=0, & t \in[0, T] \subset \mathbb{T}, \\
w^{\Delta \nabla}(t)+\zeta h(t, u(t), v(t), w(t))=0, & t \in[0, T] \subset \mathbb{T},
\end{array}
$$

subject to the boundary conditions

$$
\begin{array}{ll}
u(0)=\beta_{1} u\left(\eta_{1}\right), & u(T)=\alpha_{1} u\left(\eta_{1}\right), \\
v(0)=\beta_{2} v\left(\eta_{2}\right), & v(T)=\alpha_{2} v\left(\eta_{2}\right), \\
w(0)=\beta_{3} w\left(\eta_{3}\right), & w(T)=\alpha_{3} w\left(\eta_{3}\right),
\end{array}
$$

where $\mathcal{T}$ is a time scale and $0<\eta<T, 0<\alpha<\frac{T}{\eta}, 0<\beta<\frac{T-\alpha \eta}{T-\eta}$, and

$$
\begin{aligned}
& f(t, u, v, w)=\frac{\sqrt{t+3}(u+v+w+1)(3+\sin (v))(u+v+w)}{4,000(u+v+w)+1}, \\
& g(t, u, v, w)=\frac{\sqrt{t+1}(u+v+w+1)(4+\cos (w))(u+v+w)}{6,000(u+v+w)+1}, \\
& h(t, u, v, w)=\frac{\sqrt{t+1}(u+v+w+1)(3+\cos (u))(u+v+w)}{6,000(u+v+w)+1} .
\end{aligned}
$$

We can formulate the problem (P2) as

$$
\begin{cases}u(t)=\lambda \int_{0}^{1} k_{1}(t, s) f(s, u(s), v(s), w(s)) \nabla s, & t \in[0, T], \\ v(t)=\mu \int_{0}^{1} k_{2}(t, s) g(s, u(s), v(s), w(s)) \nabla s, & t \in[0, T] \\ w(t)=\zeta \int_{0}^{1} k_{3}(t, s) h(s, u(s), v(s), w(s)) \nabla s, & t \in[0, T]\end{cases}
$$


where

$$
\begin{aligned}
& \left\{\frac{\left(\left(1-\beta_{1}\right) t+\beta_{1} \eta_{1}\right)(T-s)}{\left(T-\alpha_{1} \eta_{1}\right)-\beta_{1}\left(T-\eta_{1}\right)}-\frac{\left(\left(\beta_{1}-\alpha_{1}\right) t-\beta_{1} T\right)\left(\eta_{1}-s\right)}{\left(T-\alpha_{1} \eta_{1}\right)-\beta_{1}\left(T-\eta_{1}\right)}-(t-s), \quad 0 \leq s \leq t \leq T, s \leq \eta_{1},\right. \\
& k_{1}(t, s)= \begin{cases}\frac{\left(\left(1-\beta_{1}\right) t+\beta_{1} \eta_{1}\right)(T-s)}{\left(T-\alpha_{1} \eta_{1}\right)-\beta_{1}\left(T-\eta_{1}\right)}-\frac{\left(\left(\beta_{1}-\alpha_{1}\right) t-\beta_{1} T\right)\left(\eta_{1}-s\right)}{\left(T-\alpha_{1} \eta_{1}\right)-\beta_{1}\left(T-\eta_{1}\right)}, & 0 \leq t \leq s \leq \eta_{1}, \\
\frac{\left(\left(1-\beta_{1}\right) t+\beta_{1} \eta_{1}\right)(T-s)}{\left(T-\alpha_{1} \eta_{1}\right)-\beta_{1}\left(T-\eta_{1}\right)}, & 0 \leq t \leq s \leq T, s \geq \eta_{1}, \\
\left.\left(1-\beta_{1}\right) t+\beta_{1}\right)(T-s)\end{cases} \\
& \frac{\left(\left(1-\beta_{1}\right) t+\beta_{1} \eta_{1}\right)(T-s)}{\left(T-\alpha_{1} \eta_{1}\right)-\beta_{1}\left(T-\eta_{1}\right)}-(t-s), \quad \eta_{1} \leq s \leq t \leq T, \\
& k_{2}(t, s)= \begin{cases}\frac{\left(\left(1-\beta_{2}\right) t+\beta_{2} \eta_{2}\right)(T-s)}{\left(T-\alpha_{2} \eta_{2}\right)-\beta_{2}\left(T-\eta_{2}\right)}-\frac{\left(\left(\beta_{2}-\alpha_{2}\right) t-\beta_{2} T\right)\left(\eta_{2}-s\right)}{\left(T-\alpha_{2} \eta_{2}\right)-\beta_{2}\left(T-\eta_{2}\right)}-(t-s), & 0 \leq s \leq t \leq T, s \leq \eta_{2}, \\
\frac{\left(\left(1-\beta_{2}\right) t+\beta_{2} \eta_{2}\right)(T-s)}{\left(T-\alpha_{2} \eta_{2}\right)-\beta_{2}\left(T-\eta_{2}\right)}-\frac{\left(\left(\beta_{2}-\alpha_{2}\right) t-\beta_{2} T\right)\left(\eta_{2}-s\right)}{\left(T-\alpha_{2} \eta_{2}\right)-\beta_{2}\left(T-\eta_{2}\right)}, & 0 \leq t \leq s \leq \eta_{2}, \\
\frac{\left(\left(1-\beta_{2}\right) t+\beta_{2} \eta_{2}\right)(T-s)}{\left(T-\alpha_{2} \eta_{2}\right)-\beta_{2}\left(T-\eta_{2}\right)}, & 0 \leq t \leq s \leq T, s \geq \eta_{2}, \\
\frac{\left(\left(1-\beta_{2}\right) t+\beta_{2} \eta_{2}\right)(T-s)}{\left(T-\alpha_{2} \eta_{2}\right)-\beta_{2}\left(T-\eta_{2}\right)}-(t-s), & \eta_{1} \leq s \leq t \leq T,\end{cases} \\
& k_{3}(t, s)= \begin{cases}\frac{\left(\left(1-\beta_{3}\right) t+\beta_{3} \eta_{3}\right)(T-s)}{\left(T-\alpha_{3} \eta_{3}\right)-\beta_{3}\left(T-\eta_{3}\right)}-\frac{\left(\left(\beta_{3}-\alpha_{3}\right) t-\beta_{3} T\right)\left(\eta_{3}-s\right)}{\left(T-\alpha_{3} \eta_{3}\right)-\beta_{3}\left(T-\eta_{3}\right)}-(t-s), & 0 \leq s \leq t \leq T, s \leq \eta_{3}, \\
\frac{\left(\left(1-\beta_{3}\right) t+\beta_{3} \eta_{3}\right)(T-s)}{\left(T-\alpha_{3} \eta_{3}\right)-\beta_{3}\left(T-\eta_{3}\right)}-\frac{\left(\left(\beta_{3}-\alpha_{3}\right) t-\beta_{3} T\right)\left(\eta_{3}-s\right)}{\left(T-\alpha_{3} \eta_{3}\right)-\beta_{3}\left(T-\eta_{3}\right)}, & 0 \leq t \leq s \leq \eta_{3}, \\
\frac{\left(\left(1-\beta_{3}\right) t+\beta_{3} \eta_{3}\right)(T-s)}{\left(T-\alpha_{3} \eta_{3}\right)-\beta_{3}\left(T-\eta_{3}\right)}, & 0 \leq t \leq s \leq T, s \geq \eta_{3}, \\
\frac{\left(\left(1-\beta_{3}\right) t+\beta_{3} \eta_{3}\right)(T-s)}{\left(T-\alpha_{3} \eta_{3}\right)-\beta_{3}\left(T-\eta_{3}\right)}-(t-s), & \eta_{1} \leq s \leq t \leq T .\end{cases}
\end{aligned}
$$

Lemma 4.1 Let $0<\alpha_{i}<\frac{\eta_{i}}{T}, 0<\beta_{i}<\frac{T-\alpha_{i} \eta_{i}}{T-\eta_{i}},(u(t), v(t), w(t))$ be a solution of the problem (P2),

then

$$
\min _{t \in[0, T]} u(t) \geq \gamma_{1} \max _{t \in[0, T]} u(t), \quad \min _{t \in[0, T]} v(t) \geq \gamma_{2} \max _{t \in[0, T]} v(t), \quad \min _{t \in[0, T]} w(t) \geq \gamma_{3} \max _{t \in[0, T]} w(t),
$$

where

$$
\gamma_{i}=\min \left\{\frac{\alpha_{i}\left(T-\eta_{i}\right)}{T-\alpha_{i} \eta_{i}}, \frac{\alpha_{i} \eta_{i}}{T}, \frac{\beta_{i}\left(T-\eta_{i}\right)}{T}, \frac{\beta_{i} T}{T}\right\}, \quad i=1,2,3
$$

We consider the case

$$
T=1, \quad \eta_{1}=\eta_{2}=\eta_{3}=\frac{1}{16}, \quad \beta_{1}=\beta_{2}=\beta_{3}=\frac{1}{3}, \quad \alpha_{1}=\alpha_{2}=\alpha_{3}=8
$$

After an easy computation, we conclude

$$
\begin{aligned}
& A=B=C=\frac{48}{27}, \quad \gamma=\frac{1}{48}, \quad \tilde{A}=\tilde{B}=\tilde{C}=\frac{65}{1,296}, \\
& f_{0}^{s}=6, \quad f_{0}^{i}=3 \sqrt{3}, \quad f_{\infty}^{s}=\frac{1}{500}, \quad f_{\infty}^{i}=\frac{\sqrt{3}}{2,000}, \\
& g_{0}^{s}=4 \sqrt{2}, \quad g_{0}^{i}=4, \quad g_{\infty}^{s}=\frac{\sqrt{2}}{1,200}, \quad g_{\infty}^{i}=\frac{1}{2,000}, \\
& h_{0}^{s}=3 \sqrt{2}, \quad h_{0}^{i}=3, \quad h_{\infty}^{s}=\frac{\sqrt{2}}{1,500}, \quad h_{\infty}^{i}=\frac{1}{3,000}, \\
& M_{1}=6, \quad M_{2}=4 \sqrt{2}, \quad M_{3}=3 \sqrt{2}, \\
& m_{1}=\frac{\sqrt{3}}{2,000}, \quad m_{2}=\frac{1}{2,000}, \quad m_{3}=\frac{1}{3,000} .
\end{aligned}
$$


Choose $a=\frac{1}{3}, b=\frac{1}{3}, c=\frac{1}{3}, d=\frac{1}{3}$. Then

$$
\begin{aligned}
& L_{1}=\frac{b}{\gamma \tilde{A} f_{0}^{i}} \approx 61.3945, \quad L_{2}=\frac{C}{A f_{\infty}^{s}} \approx 93.7500, \quad L_{3}=\frac{a}{\gamma \tilde{B} g_{0}^{i}} \approx 79.7538, \\
& L_{4}=\frac{d}{B g_{\infty}^{s}} \approx 159.0990, \quad L_{5}=\frac{1-a-b}{\gamma \tilde{C} h_{0}^{i}} \approx 106.3385, \\
& L_{6}=\frac{1-c-d}{C h_{\infty}^{s}} \approx 198.8738, \\
& \lambda_{0}=\frac{a}{A M_{1}} \approx 0.0313, \quad \mu_{0}=\frac{b}{B M_{2}} \approx 0.0331, \quad \zeta_{0}=\frac{1-a-b}{C M_{3}} \approx 0.0442, \\
& \tilde{\lambda}_{0}=\frac{c}{\gamma \tilde{A} m_{1}} \approx 368,370, \quad \tilde{\mu}_{0}=\frac{d}{\gamma \tilde{B} m_{2}} \approx 638,370, \quad \tilde{\zeta}_{0}=\frac{1-c-d}{\gamma \tilde{C} m_{3}} \approx 957,050 .
\end{aligned}
$$

Thus

(1) from Theorem 2.16, for $\lambda \in\left(L_{1}, L_{2}\right), \mu \in\left(L_{3}, L_{4}\right), \zeta \in\left(L_{5}, L_{6}\right)$, the problem (P2) has a positive solution;

(2) from Theorem 3.1, for $\lambda \in\left(0, \lambda_{0}\right), \mu \in\left(0, \mu_{0}\right), \zeta \in\left(0, \zeta_{0}\right)$, the problem (P2) has no positive solution;

(3) from Theorem 3.8, for $\lambda \in\left(\tilde{\lambda}_{0}, \infty\right), \mu \in\left(\tilde{\mu}_{0}, \infty\right), \zeta \in\left(\tilde{\zeta}_{0}, \infty\right)$ the problem (P2) has no positive solution.

\subsection{Application to system of boundary value problems of fractional differential equations}

Consider the system of nonlinear fractional differential equation (the problem (P3))

$$
\begin{array}{ll}
D_{0+}^{\alpha} u(t)+\lambda f(t, u(t), v(t), w(t))=0, & t \in(0,1), \\
D_{0+}^{\alpha} v(t)+\mu g(t, u(t), v(t), w(t))=0, & t \in(0,1), \\
D_{0+}^{\alpha} w(t)+\zeta h(t, u(t), v(t), w(t))=0, & t \in(0,1),
\end{array}
$$

subject to the boundary conditions

$$
\begin{array}{ll}
u(0)=u^{\prime}(0)=u^{\prime}(1)=0, & {\left[D_{0+}^{\delta} u(t)\right]_{t=1}=0,} \\
v(0)=v^{\prime}(0)=v^{\prime}(1)=0, & {\left[D_{0+}^{\delta} v(t)\right]_{t=1}=0,} \\
w(0)=w^{\prime}(0)=w^{\prime}(1)=0, & {\left[D_{0+}^{\delta} w(t)\right]_{t=1}=0,}
\end{array}
$$

where $\alpha=3.5, \delta=1.5$, and

$$
\begin{aligned}
& f(t, u, v, w)=(u+v+w) \frac{100(u+v+w)+1}{u+v+w+1}(3+\sin (u+v+w)), \\
& g(t, u, v, w)=(u+v+w) \frac{80(u+v+w)+1}{u+v+w+1}(4+\sin (u+v+w)), \\
& h(t, u, v, w)=(u+v+w) \frac{125(u+v+w)+1}{u+v+w+1}(3+\cos (u+v+w)) .
\end{aligned}
$$


We can check that

$$
k_{1}(t, s)=k_{2}(t, s)=k_{3}(t, s)= \begin{cases}\frac{t^{\alpha-1}(1-s)^{\alpha-\delta-1}-(t-s)^{\alpha-1}}{\Gamma(\alpha)}, & 0 \leq s \leq t \leq 1, \\ \frac{t^{\alpha-1}(1-s)^{\alpha-\delta-1}}{\Gamma(\alpha)}, & 0 \leq t \leq s \leq 1 .\end{cases}
$$

It is easy to verify that $\max _{t \in[0,1]} k_{i}(t, s)=k_{i}(1, s), i=1,2,3$, for each $s \in[0,1]$ and there is a positive constant $\gamma \in(0,1)$ such that [58]

$$
\min _{t \in\left[\frac{1}{2}, 1\right]} k_{i}(t, s) \geq \gamma k_{i}(1, s), \quad 0<s<1, i=1,2,3, \gamma=\min \left\{\frac{(1 / 2)^{\alpha-\delta-1}}{2^{\delta}-1},\left(\frac{1}{2}\right)^{\alpha-1}\right\} .
$$

Thus

$$
\begin{aligned}
& A=B=C=\int_{0}^{1} k_{i}(1, s) d s=\frac{3}{4 \Gamma(4.5)} \approx 0.0645, \quad \gamma=\left(\frac{1}{2}\right)^{2.5}, \\
& \tilde{A}=\tilde{B}=\tilde{C}=0.0114, \\
& f_{0}^{s}=3, \quad f_{0}^{i}=3, \quad f_{\infty}^{s}=400, \quad f_{\infty}^{i}=200, \\
& g_{0}^{s}=4, \quad g_{0}^{i}=4, \quad g_{\infty}^{s}=400, \quad g_{\infty}^{i}=240, \\
& h_{0}^{s}=3, \quad h_{0}^{i}=3, \quad h_{\infty}^{s}=500, \quad h_{\infty}^{i}=250, \\
& M_{1}=400, \quad M_{2}=400, \quad M_{3}=500, \quad m_{1}=3, \quad m_{2}=4, \quad m_{3}=3 .
\end{aligned}
$$

Choose $a=\frac{1}{3}, b=\frac{1}{3}, c=\frac{1}{3}, d=\frac{1}{3}, \xi=\frac{1}{2}, \eta=1$. Then

$$
\begin{array}{ll}
K_{1}=\frac{b}{\gamma \tilde{A} f_{\infty}^{i}} \approx 0.8269, & K_{2}=\frac{a}{A f_{0}^{s}} \approx 1.7227, \quad K_{3}=\frac{1-b}{\gamma \tilde{B} g_{\infty}^{i}} \approx 0.6891, \\
K_{4}=\frac{1-a}{B g_{0}^{s}} \approx 1.2920, \quad K_{5}=\frac{1-a-b}{\gamma \tilde{C} h_{\infty}^{i}} \approx 0.6615, \quad K_{6}=\frac{1-c-d}{C h_{0}^{s}} \approx 1.7227, \\
\lambda_{0}=\frac{a}{A M_{1}} \approx 0.0129, \quad \mu_{0}=\frac{b}{B M_{2}} \approx 0.0129, \quad \zeta_{0}=\frac{1-a-b}{C M_{3}} \approx 0.0103, \\
\tilde{\lambda}_{0}=\frac{c}{\gamma \tilde{A} m_{1}} \approx 55.1249, & \tilde{\mu}_{0}=\frac{d}{\gamma \tilde{B} m_{2}} \approx 41.3437, \quad \tilde{\zeta}_{0}=\frac{1-c-d}{\gamma \tilde{C} m_{3}} \approx 55.1249 .
\end{array}
$$

Thus,

(1) From Theorem 2.1, for $\lambda \in\left(K_{1}, K_{2}\right), \mu \in\left(K_{3}, K_{4}\right), \zeta \in\left(K_{5}, K_{6}\right)$, the problem (P3) has a positive solution;

(2) from Theorem 3.1, for $\lambda \in\left(0, \lambda_{0}\right), \mu \in\left(0, \mu_{0}\right), \zeta \in\left(0, \zeta_{0}\right)$, the problem (P3) has no positive solution;

(3) from Theorem 3.8, for $\lambda \in\left(\tilde{\lambda}_{0}, \infty\right), \mu \in\left(\tilde{\mu}_{0}, \infty\right), \zeta \in\left(\tilde{\zeta}_{0}, \infty\right)$ the problem (P3) has no positive solution. 


\section{Acknowledgements}

The work is sponsored by the NSFC (11201109), Anhui Provincial Natural Science Foundation (1408085QA07), the Higher School Natural Science Project of Anhui Province (KJ2014A200) and the outstanding talents plan of Anhui High school.

Received: 6 June 2016 Accepted: 23 August 2016 Published online: 18 October 2016

\section{References}

1. Anderson, R, May, R: Infectious Diseases of Humans: Dynamics and Control. Oxford University Press, Oxford (1991)

2. Song, $\mathrm{X}, \mathrm{Chen}, \mathrm{L}$ : Optimal harvesting and stability for a predator -prey system with stage structure. Acta Math. Appl. Sin. 18, 307-314 (2002)

3. Webb, J, Infante, G: Positive solutions of nonlocal boundary value problems: a unified approach. J. Lond. Math. Soc. (2) 74, 673-693 (2006)

4. Webb, J, Lan, K: Eigenvalue criteria for existence of multiple positive solutions of nonlinear boundary value problems of local and nonlocal type. Topol. Methods Nonlinear Anal. 27, 91-116 (2006)

5. Infante, G, Webb, JRL: Loss of positivity in a nonlinear scalar heat equation. NoDEA Nonlinear Differ. Equ. Appl. 13 249-261 (2006)

6. Graef, J, Yang, B: Positive solutions to a multi-point higher order boundary value problem. J. Math. Anal. Appl. 316 , 409-421 (2006)

7. Ma, R: Positive solutions of a nonlinear m-point boundary value problem. Comput. Math. Appl. 42, 755-765 (2001)

8. Ma, R, Castaneda, N: Existence of solutions of nonlinear m-point boundary-value problems. J. Math. Anal. Appl. 256, 556-567 (2001)

9. Hong, S: Triple positive solutions of three-point boundary value problems for $p$-Laplacian dynamic equations on time scales. J. Comput. Appl. Math. 206, 967-976 (2007)

10. Sun, J, Li, W: Existence of solutions to nonlinear first-order PBVPs on time scales. Nonlinear Anal. 67, $883-888$ (2007)

11. Chang, Y, Li, W: Existence results for second-order dynamic inclusion with $\mathrm{m}$-point boundary value conditions on time scales. Appl. Math. Lett. 20, 885-891 (2007)

12. Agarwal, $\mathrm{R}$, et al.: Multiple positive solutions of singular Dirichlet problems on time scales via variational methods. Nonlinear Anal. 67, 368-381 (2007)

13. Sang, Y, Su, H: Several sufficient conditions of solvability for a nonlinear higher order three-point boundary value problem on time scales. Appl. Math. Comput. 190, 566-575 (2007)

14. Zhao, J, Lian, H, Ge, W: Existence of positive solutions for nonlinear mpoint boundary value problems on time scales. Bound. Value Probl. 2012, Article ID 4 (2012)

15. Zhang, Q, He, X, Sun, H: Positive solutions for Sturm-Liouville BVPs on time scales via sub-supersolution and variational methods. Bound. Value Probl. 2013, Article ID 123 (2013)

16. Jia, M, Liu, X: Three nonnegative solutions for fractional differential equations with integral boundary conditions. Comput. Math. Appl. 62, 1405-1412 (2011)

17. Jia, M, Liu, X: Multiplicity of solutions for integral boundary value problems of fractional differential equations with upper and lower solutions. Appl. Math. Comput. 232, 313-323 (2014)

18. Kosmatov, N: A singular boundary value problem for nonlinear differential equations of fractional order. J. Appl. Math. Comput. 29, 125-135 (2009)

19. Agarwal, RP, et al.: Positive solutions for Dirichlet problems of singular nonlinear fractional differential equations J. Math. Anal. Appl. 371, 57-68 (2010)

20. Jiang, D, Yuan, C: The positive properties of the Green function for Dirichlet-type boundary value problems of nonlinear fractional differential equations and its application. Nonlinear Anal. 15, 710-719 (2010)

21. Agarwal, RP, O'Regan, D: A coupled system of difference equations. Appl. Math. Comput. 114, $39-49$ (2000)

22. $\mathrm{Wu}, \mathrm{G}, \mathrm{Li}, \mathrm{L}$, Cong, $\mathrm{X}$, Miao, X: Multiple positive solutions to multipoint boundary value problem for a system of second-order nonlinear semipositone differential equations on time scales. J. Appl. Math. 2013, Article ID 679316 (2013)

23. Wong, P, Agarwal, RP: Existence theorems for a system of difference equations with $(n, p)$-type conditions. Appl. Math. Comput. 123, 389-407 (2001)

24. Ding, $Y, X u, J$, Wei, Z: Positive solutions for a system of discrete boundary value problem. Bull. Malays. Math. Soc. 38 1207-1221 (2015)

25. Cetin, E, Topal, SS: Existence of multiple positive solutions for the system of higher order boundary value problems on time scales. Math. Comput. Model. 52, 1-11 (2010)

26. Infante, G, Minhós, F, Pietramala, P: Non-negative solutions of systems of ODEs with coupled boundary conditions. Commun. Nonlinear Sci. Numer. Simul. 17, 4952-4960 (2012)

27. Cui, Y, Zou, Y: An existence and uniqueness theorem for a second order nonlinear system with coupled integral boundary value conditions. Appl. Math. Comput. 256, 438-444 (2015)

28. Greaf, J, Kong, L, Minhós, F: Higher order boundary value problems with $\phi$-Laplacian and functional boundary conditions. Comput. Math. Appl. 61, 236-249 (2011)

29. Ahmad, N, Khan, R: Positive solutions for a class of coupled system of singular three-point boundary value problems. Bound. Value Probl. 2009, Article ID 273063 (2009)

30. Cui, Y, Zou, Y: Monotone iterative method for differential systems with coupled integral boundary value problems. Bound. Value Probl. 2013, Article ID 245 (2013)

31. Martins, E, Ferreira, W: Positive solution for a class of coupled $(p, q)$-Laplacian nonlinear systems. Bound. Value Probl. 2014, Article ID 21 (2014)

32. Ahmad, N, Talib, I, Tunc, C: Existence of solution for first-order coupled system with nonlinear coupled boundary conditions. Bound. Value Probl. 2015, Article ID 134 (2015)

33. Ma, R: Multiple nonnegative solutions of second-order systems of boundary value problems. Nonlinear Anal. 42 , 1003-1010 (2000)

34. Wang, $\mathrm{H}$ : On the number of positive solutions of nonlinear systems. J. Math. Anal. Appl. 281, 287-306 (2003)

35. Liu, W, Liu, L, Wu, Y: Positive solutions of a singular boundary value problem for systems of second-order differential equations. Appl. Math. Comput. 208, 511-519 (2009) 
36. Zhu, F, Liu, L, Wu, Y: Positive solutions for systems of a nonlinear fourth-order singular semipositone boundary value problems. Appl. Math. Comput. 216, 448-457 (2010)

37. Goodrich, C: Existence of a positive solution to a system of discrete fractional boundary value problems. Appl. Math. Comput. 217, 4740-4753 (2011)

38. Goodrich, C: Coupled systems of boundary value problems with nonlocal boundary conditions. Appl. Math. Lett. 41, 17-22 (2015)

39. Yang, W: Positive solutions for nonlinear semipositone fractional $q$-difference system with coupled integral boundary conditions. Appl. Math. Comput. 244, 702-725 (2014)

40. $\mathrm{Xu}, \mathrm{N}$ : Iterative solutions for a coupled system of fractional differential-integral equations with two-point boundary conditions. Appl. Math. Comput. 244, 903-911 (2014)

41. Su, X: Boundary value problem for a coupled system of nonlinear fractional differential equations. Appl. Math. Lett. 22, 64-69 (2009)

42. Zhang, Y, Bai, Z, Feng, T: Existence results for a coupled system of nonlinear fractional three-point boundary value problems at resonance. Comput. Math. Appl. 61, 1032-1047 (2011)

43. Rehman, $M$, Khan, R: A note on boundary value problems for a coupled system of fractional differential equations, Comput. Math. Appl. 61, 2630-2637 (2011)

44. Yang, W: Positive solutions for a coupled system of nonlinear fractional differential equations with integral boundary conditions. Comput. Math. Appl. 63, 288-297 (2012)

45. Yuan, C: Two positive solutions for $(n-1,1)$-type semipositone integral boundary value problems for coupled systems of nonlinear fractional differential equation. Commun. Nonlinear Sci. Numer. Simul. 17, 930-942 (2012)

46. Jiang, W: Solvability for a coupled system of fractional differential equations at resonance. Nonlinear Anal., Real World Appl. 13, 2285-2292 (2012)

47. $\mathrm{Hu}, \mathrm{Z}, \mathrm{Liu}, \mathrm{W}, \mathrm{Chen}, \mathrm{T}$ : Existence of solutions for a coupled system of fractional differential equations at resonance. Bound. Value Probl. 2012, Article ID 98 (2012)

48. Zhang, X, Zhu, C, Wu, Z: Solvability for a coupled system of fractional differential equations with impulses at resonance. Bound. Value Probl. 2013, Article ID 80 (2013)

49. Liu, Y, Nieto, J, Otero-Zarraquiños, Ó: Existence results for a coupled system of nonlinear singular fractional differential equations with impulse effects. Math. Probl. Eng. 2013, Article ID 498781 (2013)

50. Henderson, J, Luca, R: Nonexistence of positive solutions for a system of coupled fractional boundary value problems. Bound. Value Probl. 2015, Article ID 138 (2015)

51. Henderson, J, Luca, R: Positive solutions for a system of fractional differential equations with coupled integral boundary conditions. Appl. Math. Comput. 249, 182-197 (2014)

52. Wang, G, Ahmad, B, Zhang, L: A coupled system of nonlinear fractional differential equations with multipoint fractional boundary conditions on an unbounded domain. Abstr. Appl. Anal. 2012, Article ID 248709 (2012)

53. Jiang, J: Solvability of anti-periodic boundary value problem for coupled system of fractional $p$-Laplacian equation. Adv. Differ. Equ. 2015, Article ID 305 (2015)

54. Liu, Z, Sun, J: Nonlinear boundary value problems of fractional differential systems. Comput. Math. Appl. 64, 463-475 (2012)

55. Guo, D, Lakshmikantham, V: Nonlinear Problems in Abstract Cones. Academic Press, San Diego (1988)

56. Wang, H: On the number of positive solutions of nonlinear systems. J. Math. Anal. Appl. 281, 287-306 (2003)

57. Il'in, VA, Moiseev, El: Nonlocal boundary value problem of the first kind for a Sturm-Liouville operator in its differential and finite difference aspects. Differ. Equ. 23, 803-810 (1987)

58. I'in, VA, Moiseev, El: Nonlocal boundary value problem of the second kind for a Sturm-Liouville operator. Differ. Equ. 23, 979-987 (1987)

59. Goodrich, C: Existence of a positive solution to a class of fractional differential equations. Appl. Math. Lett. 23, 1050-1055 (2010)

\section{Submit your manuscript to a SpringerOpen ${ }^{\ominus}$ journal and benefit from:}

- Convenient online submission

- Rigorous peer review

- Immediate publication on acceptance

- Open access: articles freely available online

- High visibility within the field

- Retaining the copyright to your article 